\title{
Covariant model for Dalitz decays of decuplet baryons to octet baryons
}

\author{
G. Ramalho® \\ Laboratório de Física Teórica e Computacional-LFTC, Universidade Cruzeiro do Sul and Universidade \\ Cidade de São Paulo, 01506-000 São Paulo, SP, Brazil
}

(Received 2 March 2020; accepted 11 August 2020; published 15 September 2020)

\begin{abstract}
In the last years it became possible to measure in HADES the dilepton decays of several baryons. The baryon dilepton decays provide information about the electromagnetic structure of the baryons in the timelike region. In the present work, we study the $B^{\prime} \rightarrow e^{+} e^{-} B$ decays, where $B^{\prime}$ is a baryon decuplet member and $B$ is a baryon octet member. Our calculations are based on the covariant spectator quark model, where the contribution of the quark core is complemented with an $S U(3)$ contribution from the pion cloud. The pion cloud contribution prove to be relevant in the range of study. We present predictions for the $\Sigma^{0}(1385) \rightarrow e^{+} e^{-} \Lambda(1116)$ and $\Sigma^{+}(1385) \rightarrow e^{+} e^{-} \Sigma^{+}(1193)$ decays, which may be tested at HADES in a near future. Predictions for the remaining decuplet baryon Dalitz decays are also presented. We conclude that different orders of magnitudes are expected for the baryon decuplet Dalitz decay widths, according to the quark content of the baryons. We also conclude that the dependence of the transition form factors on the square momentum transfer $\left(q^{2}\right)$ is important for some transitions.
\end{abstract}

DOI: 10.1103/PhysRevD.102.054016

\section{INTRODUCTION}

In the last decades, there was a significant progress in the study of the electromagnetic structure of the nucleon $(N)$ and nucleon excitations $\left(N^{*}\right)$ [1-3]. Most of the measured data were obtained through the scattering of electrons on nucleon targets $\left(e^{-} N \rightarrow e^{-} N^{*}\right)$, which probes the region where the square four-momentum transfer $q^{2}$ is negative $\left(q^{2}<0\right)$, also known as the spacelike region. In the electron scattering experiments the analysis of the data is based on the $\gamma^{*} N \rightarrow N^{*}$ transition, where the spacelike virtual photon is produced by the incoming electron, and the $\gamma^{*} N \rightarrow N^{*}$ transition form factors are extracted from the experimental cross sections. Experiments based on electron-nucleon scattering have been performed in facilities such as Jefferson Lab, MIT-Bates, ELSA, MAMI among others, to probe the electromagnetic structure of $N^{*}$ states in the first three resonance regions [1-6].

The electron scattering technique can also be used to probe the electromagnetic structure of the hyperons (baryons with strange quarks) based on the $\gamma^{*} B \rightarrow B^{\prime}$ transitions, where $B$ and $B^{\prime}$ are generic hyperons. In practice, however, the technique is almost exclusively limited to nucleon targets, since hyperons targets are difficult to produce due to their short lifetime, except in the limit $q^{2}=0$. In that

Published by the American Physical Society under the terms of the Creative Commons Attribution 4.0 International license. Further distribution of this work must maintain attribution to the author(s) and the published article's title, journal citation, and DOI. Funded by SCOAP . limit, there are measurements of magnetic moments of a few hyperons and some magnetic transition moments [6-13]. Another limitation of the electron scattering technique is that it is restricted to the $q^{2} \leq 0$ region.

The timelike region $\left(q^{2}>0\right)$ can be accessed at HADES (GSI) through some exclusive reaction channels in protonproton $(p p)$ collisions or by pion-induced reactions [14-28]. In the proton-proton collisions the channel $p p \rightarrow$ $p p e^{+} e^{-}$probes the structure of the intermediate $N^{*}$ states through the elementary reactions $N^{*} \rightarrow p \gamma^{*} \rightarrow p e^{+} e^{-}$ $[14,15,29]$. The $\Delta(1232)$ Dalitz decay was recently analyzed at HADES based on the study of the $p p \rightarrow p p e^{+} e^{-}$ channel on $p p$ scattering $[15,16]$. The results were compared our estimates [30]. The pion-induced reactions, are particularly important to study $N^{\star}$ resonances which decay into two or more pions [20,21,23-25]. Measurements of the $N(1520)$ and $N(1535)$ Dalitz decays are in progress at HADES [20,23,31-34]. In both methods, we access the region $4 m_{e}^{2} \leq q^{2} \leq\left(M_{B^{\prime}}-M_{N}\right)^{2}$, where $m_{e}$ is the electron mass, and $M_{B^{\prime}}$ and $M_{N}$ are the $N^{*}$ and nucleon masses, respectively. The production of timelike photons is clearly identified by the detection of $e^{+} e^{-}$pairs (dileptons) ${ }^{1}$ in the final state, due to the conversion $\gamma^{*} \rightarrow e^{+} e^{-}$. Experiments at HADES complement then the experiments based on electron-nucleon scattering, in the spacelike region $\left(q^{2} \leq 0\right)[18,35]$.

\footnotetext{
${ }^{1}$ Although the term dilepton can be used for muon pairs $\left(\mu^{+} \mu^{-}\right)$, we follow the usual nomenclature and use dilepton to refer to a electron-positron pair $\left(e^{+} e^{-}\right)$.
} 
Another timelike subregion, not discussed in the present work, is the region probed by $e^{+} e^{-}$and $p \bar{p}$ collisions at $B A B A R$, BES-III, CLEO, and PANDA/FAIR [36-40], which access the baryon $(B)$ elastic form factors when $q^{2} \geq 4 M_{B}^{2}$, where $M_{B}$ is the baryon mass [7,41].

HADES provides a unique opportunity to explore the electromagnetic structure of baryons based on the $B^{\prime} \rightarrow \gamma^{*} B$ transitions, where $B^{\prime}$ and $B$ are generic baryons, through the dilepton decays $\left(B^{\prime} \rightarrow e^{+} e^{-} B\right)$ [14,15,18,20,23-25]. Different from the traditional electron-nucleon scattering, at HADES one can probe the electromagnetic structure of the hyperons in the kinematic region $4 m_{e}^{2} \leq q^{2} \leq\left(M_{B^{\prime}}-M_{B}\right)^{2}$, where $M_{B^{\prime}}, M_{B}$ are the baryon masses [25,35,42]. Measurements of strangeness production are possible due to the large acceptance and excellent particle identification, including dileptons in the final state [17]. In progress are feasibility studies on the $\Sigma(1385), \Lambda(1404)$ and $\Lambda(1520)$ Dalitz decays by the HADES collaboration [42-44]. Those studies suggest that those decays can be measured at GSI in the next few years and subsequently also at FAIR $[18,20,42,45,46]$.

From the theoretical side there are not many models available for baryon electromagnetic transitions in the timelike region [19,26-28,45,47-51]. An important constraint on those models is that the transition between the spacelike region and the timelike region (interval between $q^{2}=0$ and $q^{2}=4 m_{e}^{2}$ ) must be smooth [18,23,35]. There are a few theoretical issues, which need to be discussed: What happens in the transition between the spacelike region $\left(q^{2} \leq 0\right)$, and the timelike region $\left(q^{2}>0\right)$ where relevant imaginary components appear on the transition form factors above the two-pion threshold $\left(q^{2}>4 m_{\pi}^{2}\right)$ for isovector transitions, and above the three-pion threshold $\left(q^{2}>9 m_{\pi}^{2}\right)$ for isoscalar transitions ( $m_{\pi}$ is the pion mass). How important are the physical poles associated with the meson resonances. How significant is the $q^{2}$ dependence of the form factors, and how are form factors modified near the pseudothreshold $q^{2}=\left(M_{B^{\prime}}-M_{B}\right)^{2}$ [15,29,52-55].

In the spacelike region, including the limit $Q^{2}=0$, there are calculations based on nonrelativistic and relativistic quark models [47,56-60], Dyson-Schwinger equations [61,62], lattice QCD simulations [63], QCD sum rules $[64,65]$, Skyrme and soliton models [66-68], chiral perturbation theory and large $N_{c}$ limit [46,69-71].

From the analysis of the spacelike data, one can conclude that models based strictly on the quark degrees of freedom are insufficient to explain the measured transition form factors. The effects associated with the meson cloud dressing of the bare cores are crucial to describe the data in the region $0 \geq q^{2}>-2 \mathrm{GeV}^{2}$, as demonstrated already for the $\Delta(1332)[1-4,72-74]$. Our model for the $\Delta(1232)$ Dalitz decay [30], which describe the HADES data [15], corroborates also the importance of the of pion cloud for the $\gamma^{*} N \rightarrow \Delta(1232)$ transition in the timelike region, as in the spacelike region. There is therefore a great interest in studying the roles of the valence quark and meson cloud effects in the timelike region [3,30-32,75].

Motivated by the experiments planned for HADES, in the present work we focus on the $B^{\prime} \rightarrow \gamma^{*} B$ transitions, where $B^{\prime}$ is a baryon decuplet member and $B$ is a baryon octet member (decuplet baryon decays). We restrict for now our study to baryon systems that best fit an $S U(3)$ quark model classification (baryon octet and baryon decuplet). Our calculations are based on the covariant spectator quark model $[3,76,77]$ developed previously for the $\gamma^{*} B \rightarrow B^{\prime}$ transitions in the spacelike region [13].

The covariant spectator quark model provides an alternative to valence quark models which do not take into account meson cloud excitations of the bare cores, and simplified vector meson dominance (VMD) models [19,49-51], which do not take into account the underlying quark substructure of the baryons. The formalism has been used in the study of the electromagnetic and the axial structure of the nucleon, several nucleon excitations, and hyperons [7-11,30-32,72-86].

The covariant spectator quark model of the $\gamma^{*} B \rightarrow B^{\prime}$ transition [13] is extended in the present work to the timelike region. Within the formalism, the octet baryon to decuplet baryon electromagnetic transitions are dominated by the magnetic transition form factor $[3,76,77]$, which can be decomposed into valence quark and meson cloud contributions [12,13]. The meson cloud contribution is calculated from a microscopic pion-baryon model, calibrated by the $\gamma^{*} N \rightarrow \Delta(1232)$ transition, and extended to the octet baryon to decuplet baryon electromagnetic transitions [12,13,30].

We use our formalism to estimate the baryon decuplet Dalitz decay widths in terms of the square invariant mass of the dilepton pair $q^{2}$, and the square invariant energy $W^{2}$ of the $\gamma^{*} B$ system [14,29]. We present, in particular, predictions for the $\Sigma^{0}(1385) \rightarrow e^{+} e^{-} \Lambda(1116)$ and $\Sigma^{+}(1385) \rightarrow$ $e^{+} e^{-\Sigma^{+}}(1193)$ decays, which may be tested by future HADES experiments [18,42]. As for the remaining decays, we estimate that the magnitudes of the $\Xi^{0}(1530) \rightarrow$ $e^{+} e^{-} \Xi^{0}(1318)$ and $\Sigma^{+}(1385) \rightarrow e^{+} e^{-\Sigma^{+}}(1193)$ decay widths are comparable to the magnitude of the $\Delta(1232) \rightarrow$ $e^{+} e^{-} N$ decay width, as suggested by $S U(3)$ and $U$-spin estimates $[13,59]$. We present also calculations for the radiative decay widths in terms of the invariant mass $W$, and compare our estimates with the available data. We conclude also that our estimate of the $\Sigma^{-}(1385) \rightarrow$ $\gamma \Sigma^{-}(1193)$ width, unknown at the moment, is close to the present experimental limit, and may therefore be measured in a near future.

This article is organized as follows: In the next section, we review the formalism associated with the radiative and Dalitz decays of $3 / 2^{+}$baryons into $1 / 2^{+}$baryons. The covariant spectator quark model is discussed in Sec. III, where we present also numerical results for the transition form factors. Our results for the radiative and Dalitz decays 
of the decuplet baryons $B^{\prime}$ are presented and discussed in Sec. IV. The outlook and conclusions are presented in Sec. V.

\section{DALITZ DECAY OF DECUPLET BARYONS}

A baryon $B^{\prime}$ can decay in different channels, including meson-baryon states, (multi-meson)-baryon states, the radiative decay $(\gamma B)$ and the dilepton decay $\left(e^{+} e^{-} B\right)$. In the present section, we focus on the radiative $\left(B^{\prime} \rightarrow \gamma B\right)$ and dilepton $\left(B^{\prime} \rightarrow e^{+} e^{-} B\right)$ decays. The formalism described below is a generalization of the formalism for the $\Delta(1232) \rightarrow$ $\gamma N$ and $\Delta(1232) \rightarrow e^{+} e^{-} N$ decays $[29,30,87,88]$.

We assume that $B^{\prime}$ is a member of the baryon decuplet (state $\frac{3}{2}^{+}$) and that $B$ a is a member of the baryon octet (state $\frac{1}{2}^{+}$). Both baryons have positive parity. As before, $M_{B^{\prime}}$ and $M_{B}$ represent the mass of $B^{\prime}$ and $B$, respectively.

The Dalitz decay of the baryon $B^{\prime}$ is determined by the function $\Gamma_{\gamma^{*} B}(q, W)$, where $W$ is the energy of the resonance $B^{\prime}, q=\sqrt{q^{2}}$ and $q^{2}$ is the virtual photon $\left(\gamma^{*}\right)$ square four-momentum. The baryon $B^{\prime}$ Dalitz decay is the consequence of the decay of the timelike virtual photon into a pair of electrons $\left(\gamma^{*} \rightarrow e^{+} e^{-}\right)$.

The function $\Gamma_{\gamma^{*} B}(q, W)$ can be written $[29,30,75,87]$ as

$\Gamma_{\gamma^{*} B}(q, W)=\frac{\alpha}{16} \frac{\left(W+M_{B}\right)^{2}}{W^{3} M_{B}^{2}} \sqrt{y_{+} y_{-}} y_{-}\left|G_{T}\left(q^{2}, W\right)\right|^{2}$,

where $\alpha \simeq 1 / 137$ is the fine-structure constant, and

$$
y_{ \pm}=\left(W \pm M_{B}\right)^{2}-q^{2} .
$$

The function $\left|G_{T}\left(q^{2}, W\right)\right|$ depends on the Jones-Scadron form factors: $G_{M}$ (magnetic dipole), $G_{E}$ (electric quadrupole) and $G_{C}$ (Coulomb quadrupole) $[89,90]$, and takes the form

$$
\begin{aligned}
& \left|G_{T}\left(q^{2}, W\right)\right|^{2} \\
& \quad=\left|G_{M}\left(q^{2}, W\right)\right|^{2}+3\left|G_{E}\left(q^{2}, W\right)\right|^{2}+\frac{q^{2}}{2 W}\left|G_{C}\left(q^{2}, W\right)\right|^{2} .
\end{aligned}
$$

The functions $\Gamma_{\gamma B}(W)$ and $\Gamma_{e^{+} e^{-} B}(W)$ which quantify the radiative and Dalitz decays, respectively, are calculated with the assistance of the function $\Gamma_{\gamma^{*} B}(q, W)$, as discussed below.

The photon decay width is defined by the limit $q^{2}=0$ $[29,88]$

$$
\Gamma_{\gamma B}(W)=\Gamma_{\gamma^{*} B}(0, W) .
$$

The Dalitz decay width $\Gamma_{e^{+} e^{-} B}(W)$ is determined by integrating

$$
\Gamma_{e^{+} e^{-} B}^{\prime}(q, W) \equiv \frac{d \Gamma_{e^{+} e^{-} B}}{d q}(q, W),
$$

according to

$$
\Gamma_{e^{+} e^{-B}}(W)=\int_{2 m_{e}}^{W-M_{B}} \Gamma_{e^{+} e^{-} B}^{\prime}(q, W) d q .
$$

In the previous equation the interval of integration $4 m_{e}^{2} \leq$ $q^{2} \leq\left(W-M_{B}\right)^{2}$ is the consequence of the threshold of the dilepton production and the maximum value of the photon square four-momentum allowed by the $B^{\prime} \rightarrow \gamma^{*} B$ decay: $q^{2}=\left(W-M_{B}\right)^{2}$. This is the value of $q^{2}$ obtained when the photon three-momentum vanishes $|\mathbf{q}|=0[52-54,75]$. The function $\Gamma_{e^{+} e^{-} B}^{\prime}(q, W)$ can be evaluated using [15,29,30,88]

$$
\Gamma_{e^{+} e^{-B}}^{\prime}(q, W)=\frac{2 \alpha}{3 \pi q} \Gamma_{\gamma^{*} B}(q, W) .
$$

The relations (2.4), (2.6) and (2.7) demonstrate that the decay widths $\Gamma_{\gamma B}(W)$ and $\Gamma_{e^{+} e^{-} B}(W)$ are determined, once one has a model for the effective form factor $\left|G_{T}\left(q^{2}, W\right)\right|$. Note, however, that the model should be defined for arbitrary values of $W$ (invariant energy of the $\gamma^{*} B$ system), since the measurements are performed for values of $W$ which may differ from the decuplet baryon mass $\left(M_{B^{\prime}}\right)$. Our model for $\left|G_{T}\left(q^{2}, W\right)\right|$ is described in the next section.

The baryon $B^{\prime}$ radiative decay $\left(B^{\prime} \rightarrow \gamma B\right)$ measured in the experiments, correspond to the result from Eq. (2.1) in the limits $W=M_{B^{\prime}}$ and $q^{2}=0$ :

$$
\Gamma_{\gamma B} \equiv \Gamma_{\gamma^{*} B}\left(0, M_{B^{\prime}}\right) .
$$

\section{COVARIANT SPECTATOR QUARK MODEL}

In the present section, we describe the formalism associated with the covariant spectator quark model $[3,76,77]$. The covariant spectator quark model was derived from the covariant spectator theory $[76,91]$. In this framework a baryon is described as a three-constituent quark system, where a quark is free to interact with the electromagnetic fields. Integrating over the internal degrees of freedom of the noninteracting quark-pair, one reduces the three-quark system to a quark-diquark system where the spectator quark-pair is represented by an on-mass-shell diquark with an effective mass $m_{D}$ [76-78]. The effective quark-diquark wave function is free of singularities and include the quark confinement implicitly $[1,3,76,91]$. The wave functions of the baryons are built according to the spin-flavor-radial symmetries with the radial wave functions determined phenomenologically by the experimental data, or lattice QCD data for some ground state systems $[3,74,76,77]$.

In the electromagnetic interaction with the quarks, we take into account the structure associated with gluon and 
TABLE I. Mixed antisymmetric $\left|M_{A}\right\rangle$ and mixed symmetric $\left|M_{S}\right\rangle$ flavor states for the octet baryons [9,12].

\begin{tabular}{llc}
\hline \hline$B$ & \multicolumn{1}{c}{$\left|M_{A}\right\rangle$} & $\left|M_{S}\right\rangle$ \\
\hline$p$ & $\frac{1}{\sqrt{2}}(u d-d u) u$ & $\frac{1}{\sqrt{6}}[(u d+d u) u-2 u u d]$ \\
$n$ & $\frac{1}{\sqrt{2}}(u d-d u) d$ & $-\frac{1}{\sqrt{6}}[(u d+d u) d-2 d d u]$ \\
$\Lambda^{0}$ & $\frac{1}{\sqrt{12}}[s(d u-u d)-(d s u-u s d)-2(d u-d u) s]$ & $\frac{1}{2}[(d s u-u s d)+s(d u-u d)]$ \\
$\Sigma^{+}$ & $\frac{1}{\sqrt{2}}(u s-s u) u$ & $\frac{1}{\sqrt{6}}[(u s+s u) u-2 u u s]$ \\
$\Sigma^{0}$ & $\frac{1}{2}[(d s u+u s d)-s(u d+d u)]$ & $\frac{1}{\sqrt{6}}[(s d+d s) d-2 d d s]$ \\
$\Sigma^{-}$ & $\frac{1}{\sqrt{2}}(d s-s d) d$ & $-\frac{1}{\sqrt{6}}[(u d+d u) s-2 s s u]$ \\
$\Xi^{0}$ & $\frac{1}{\sqrt{2}}(u s-s u) s$ & $-\frac{1}{\sqrt{6}}[(d s+s d) s-2 s s d]$ \\
$\Xi^{-}$ & $\frac{1}{\sqrt{2}}(d s-s d) s$ & \\
\hline \hline
\end{tabular}

quark-antiquark dressing of the quarks. This structure is parametrized in terms of constituent quark electromagnetic form factors [76,77].

The covariant spectator quark model was already applied to the study of the electromagnetic structure of several baryons in the spacelike region $[9,11,72,73,78-85]$, in the timelike region $[7,30-32,75]$, to the structure of baryons in the nuclear medium [10], and to the lattice QCD regime $[74,77,86]$. We discuss next the formalism associated with the octet and decuplet baryons.

\section{A. Formalism}

In the covariant spectator quark model the baryon wave functions $\Psi_{B}(P, k)$ depend on the baryon $(P)$ and diquark (k) momenta, as well as the flavor and spin projection indices. Spin projection indices in the wave functions are suppressed for simplicity.

The wave functions of the octet baryon and the decuplet are constructed conveniently by the symmetrized states of the diquark (12), and the off-mass-shell quark (3) [76-78].

The octet baryon $B$ wave functions can be expressed, in the $S$-wave approximation as $[10,12]$

$$
\Psi_{B}(P, k)=\frac{1}{\sqrt{2}}\left[\phi_{S}^{0}\left|M_{A}\right\rangle+\phi_{S}^{1}\left|M_{S}\right\rangle\right] \psi_{B}(P, k)
$$

where $\phi_{S}^{0,1}$ are the spin-0 and spin-1 diquark components of the wave functions, $\left|M_{A}\right\rangle$ and $\left|M_{S}\right\rangle$ are the mixed antisymmetric and mixed symmetric flavor states, and $\psi_{B}(P, k)$ is the radial wave function. The explicit expressions for $\phi_{S}^{0,1}$ are presented in Refs. [10,12]. The octet baryon flavor wave functions, are presented in Table I.

The decuplet baryon $B^{\prime}$ wave functions, in the $S$-wave approximation takes the form [77]

$$
\Psi_{B^{\prime}}(P, k)=-\psi_{B^{\prime}}(P, k)\left|B^{\prime}\right\rangle \varepsilon_{P}^{\alpha}(\lambda) u_{\alpha}(P),
$$

where $u_{\alpha}(P)$ is the Rarita-Schwinger vector spin, $\psi_{B^{\prime}}(P, k)$ is the radial wave function, $\varepsilon_{P}^{\alpha}(\lambda)$ is the polarization state of the spin-1 diquark (polarization $\lambda$ ), and $\left|B^{\prime}\right\rangle$ is the flavor wave function, displayed in Table II. For a more detailed description of the polarization states $\varepsilon_{P}^{\alpha}(\lambda)$ check Refs. [72,73,76].

The radial wave functions $\psi_{B}(P, k)$ can be parametrized in terms of the variable

$$
\chi_{B}=\frac{\left(M_{B}-m_{B}\right)^{2}-(P-k)^{2}}{M_{B} m_{D}} .
$$

The representation of $\psi_{B}(P, k)$ in terms of the single variable $\chi_{B}$ is possible because the baryon $B$ and the diquark are both on-mass-shell [76].

The $\gamma^{*} B \rightarrow B^{\prime}$ transition current in relativistic impulse approximation takes the form [76-78]

$$
J^{\mu}=3 \sum_{\Gamma} \int_{k} \bar{\Psi}_{B^{\prime}}\left(P_{+}, k\right) j_{q}^{\mu} \Psi_{B}\left(P_{-}, k\right),
$$

where $P_{+}\left(P_{-}\right)$is the final (initial) baryon momentum, $k$ is the diquark momentum (on-mass-shell), and $j_{q}^{\mu}\left(q^{2}\right)$ is the quark current operator, depending on momentum transfer $q=P_{+}-P_{-}[3,72,76]$. The integration symbol represents the covariant integration in $k$, and the sum is over the diquark polarization states, including the scalar and vector components. The factor 3 takes into account the sum in the quarks based on the wave function symmetries.

The quark current $j_{q}^{\mu}$, where $q=u, d, s$, includes the electromagnetic structure of the constituent quark (gluon and quark-antiquark dressing effects) $[76,77]$. The quark current operator is represented in the form [77]

$$
j_{q}^{\mu}(q)=j_{1} \gamma^{\mu}+j_{2} \frac{i \sigma^{\mu \nu} q_{\nu}}{2 M_{N}},
$$


TABLE II. Quark flavor wave functions $\left|B^{\prime}\right\rangle$ for the decuplet baryons [77]. Not included here are the $\Delta^{++}, \Delta^{-}$and $\Omega^{-}$states.

\begin{tabular}{cc}
\hline \hline$B^{\prime}$ & $\left|B^{\prime}\right\rangle$ \\
\hline$\Delta^{+}$ & $\frac{1}{\sqrt{3}}[u u d+u d u+d u u]$ \\
$\Delta^{0}$ & $\frac{1}{\sqrt{3}}[d d u+d u d+u d d]$ \\
$\Sigma^{*+}$ & $\frac{1}{\sqrt{3}}[u u s+u s u+s u u]$ \\
$\Sigma^{* 0}$ & $\frac{1}{\sqrt{6}}[u d s+d u s+u s d+s u d+d s u+s d u]$ \\
$\Sigma^{*-}$ & $\frac{1}{\sqrt{3}}[d d s+d s d+s d d]$ \\
$\Xi^{* 0}$ & $\frac{1}{\sqrt{3}}[u s s+s u s+s s u]$ \\
$\Xi^{*-}$ & $\frac{1}{\sqrt{3}}[d s s+s d s+s s d]$ \\
\hline \hline
\end{tabular}

where $j_{i}(i=1,2)$ are the Dirac and Pauli flavor operators, acting on the third quark component of the wave function, and $M_{N}$ is the nucleon mass, as before.

The components of the quark current $j_{i}(i=1,2)$ can be decomposed as the sum of operators

$j_{i}\left(Q^{2}\right)=\frac{1}{6} f_{i+}\left(Q^{2}\right) \lambda_{0}+\frac{1}{2} f_{i-}\left(Q^{2}\right) \lambda_{3}+\frac{1}{6} f_{i 0}\left(Q^{2}\right) \lambda_{s}$,

where

$$
\begin{array}{ll}
\lambda_{0}=\left(\begin{array}{lll}
1 & 0 & 0 \\
0 & 1 & 0 \\
0 & 0 & 0
\end{array}\right), & \lambda_{3}=\left(\begin{array}{ccc}
1 & 0 & 0 \\
0 & -1 & 0 \\
0 & 0 & 0
\end{array}\right), \\
\lambda_{s}=\left(\begin{array}{ccc}
0 & 0 & 0 \\
0 & 0 & 0 \\
0 & 0 & -2
\end{array}\right), &
\end{array}
$$

are the flavor operators. These operators act on the quark wave function in flavor space, $q=(u d s)^{T}$.

The functions $f_{i+}, f_{i-}(i=1,2)$ represent the quark isoscalar and isovector form factors, respectively, based on the combinations of the quarks $u$ and $d$. The functions $f_{i 0}(i=1,2)$ represent the structure associated with the strange quark.

The explicit form for the quark form factors is included in Appendix A. For the present discussion, the relevant part is that the quark form factors are represented in terms the vector meson mass poles associated with the mesons $\rho$, $\omega$ and $\phi$ depending of the type $(l= \pm, 0)$. The expressions of the quarks form factors are valid for the spacelike and timelike regions. In the timelike region, however, the vector mass poles are corrected by finite decay widths. The isovector transitions, like $\gamma^{*} N \rightarrow \Delta(1232)$ and $\gamma^{*} \Lambda(1116) \rightarrow \Sigma^{0}(1385)$, depend on the isovector form factors (meson $\rho$ ). Other transitions depend on a combination of isovector, isoscalar and strange quark form factors.
Even though our quarks have structure, including processes which can be interpreted as meson cloud dressing of the quarks, there are processes involving the meson cloud dressing that are not taken explicitly into account. The processes in which there is a meson exchange between the different quarks cannot be represented by the quark dressing due to the meson cloud. Instead, the processes in which the meson is exchanged between different quarks are regarded in our model, as the meson is emitted and absorbed by baryon states, based on a baryon-meson molecular picture $[12,13,30]$. Those effects are discussed in more detail in Sec. III C.

We consider here the covariant spectator quark model for the $\gamma^{*} B \rightarrow B^{\prime}$ transition from Refs. [10,12,13]. As mentioned, we assume in first approximation that the octet baryon $\left(\Psi_{B}\right)$ and the decuplet baryon $\left(\Psi_{B^{\prime}}\right)$ wave functions are both described by the dominant $S$-wave quark-diquark configuration. In the transition, only the symmetric flavor components of the octet baryon wave functions $\left(\left|M_{S}\right\rangle\right)$ contribute to the transition form factors, because the decuplet baryon has no contributions from scalar diquarks. The explicit expressions are presented in Refs. [9,10,12,77]. In the $S$-wave approximation, the transition is dominated by the magnetic dipole form factor, $G_{M}$ as in the $\gamma^{*} N \rightarrow \Delta(1232)$ transition $\left(G_{E}=G_{C} \equiv 0\right)$. As a consequence, in Eq. (2.1) we can replace $\left|G_{T}\left(q^{2}, W\right)\right|$ by $\left|G_{M}\left(q^{2}, W\right)\right|$.

When we take into account the pion cloud effects, one can decompose $G_{M}$ into two components $[30,72,86]$

$$
G_{M}\left(q^{2} ; W\right)=G_{M}^{\mathrm{B}}\left(q^{2}, W\right)+G_{M}^{\pi}\left(q^{2} ; W\right),
$$

where $G_{M}^{\mathrm{B}}$ represent the contribution from the three-quark core (bare contribution) and $G_{M}^{\pi}$ represent the contribution from the pion cloud. In the previous equation, we use $q^{2}=$ $-Q^{2}$ to convert the spacelike relations for $G_{M}^{\mathrm{B}}$ and $G_{M}^{\pi}$ to the timelike region, and use $W$ to generalize the dependence of the form factor on the resonance mass $\left(M_{B^{\prime}}\right.$ in the spacelike expressions). We omit the indices $B$ and $B^{\prime}$ in the form factors for simplicity. In some octet baryon to decuplet baryon electromagnetic transitions, the contributions of the kaon cloud may be also considered. For a discussion of the magnitude of the kaon cloud contributions check Ref. [13].

It is worth noticing that the dominance of the magnetic dipole form factor is an approximation, and a consequence of the $S$-wave quark-diquark structure. In the case of the $\gamma^{*} N \rightarrow \Delta(1232)$ transition there is evidence that the quadrupole form factors $G_{E}$ and $G_{C}$ may have significant pion cloud contributions $[53,55]$. The contributions of those form factors to $\left|G_{T}\left(q^{2}, W\right)\right|$ from Eq. (2.3) are, however, not significant, since $G_{E}$ is very small and $G_{C}$ is suppressed for small $q^{2}$.

The valence quark contribution $G_{M}^{\mathrm{B}}$ and the pion cloud contribution $G_{M}^{\pi}$ are discussed in the two next subsections. The numerical results for transition form factors are 
presented afterwards. We anticipate here that as in the case of the $\gamma^{*} N \rightarrow \Delta(1232)$ transition, the pion cloud/meson cloud contributions are relevant for the description of the $\gamma^{*} B \rightarrow B^{\prime}$ transitions.

\section{B. Valence quark contributions}

The contributions from the valence quarks to the octet baryon to decuplet baryon electromagnetic form factors $\left(\gamma^{*} B \rightarrow B^{\prime}\right)$ were calculated in previous works. The expression for the magnetic form factor can be written as [12]

$$
G_{M}^{\mathrm{B}}\left(q^{2}, W\right)=\frac{4}{3 \sqrt{3}} g_{v} \mathcal{I}\left(q^{2}, W\right),
$$

where

$$
\mathcal{I}\left(q^{2}, W\right)=\int_{k} \psi_{B^{\prime}}\left(P_{+}, k\right) \psi_{B}\left(P_{-}, k\right)
$$

is the overlap integral of the octet baryon and decuplet baryon radial wave functions, and

$$
g_{v}=\frac{1}{\sqrt{2}}\left[\frac{2 M_{B}}{W+M_{B}} j_{1}^{S}\left(q^{2}\right)+\frac{M_{B}}{M_{N}} j_{2}^{S}\left(q^{2}\right)\right] .
$$

The functions $j_{i}^{S}$ represent the projection of the flavor operators into the flavor components of the decuplet baryon and the mixed symmetric component of the octet baryon flavor state [12]. The explicit expressions in terms of the quark form factors are presented in Table III.

In Table III and along the draft, we use the asterisk $\left({ }^{*}\right)$ to represent the excited states of $\Sigma$ and $\Xi$, members of the baryon decuplet. The label $\gamma^{*} N \rightarrow \Delta$ includes the $\gamma^{*} p \rightarrow \Delta^{+}$and $\gamma^{*} n \rightarrow \Delta^{0}$ transitions ( $n$ is the neutron).

The overlap integral (3.10) is invariant and can be evaluated in any frame. For convenience we use the baryon $B^{\prime}$ rest frame, where $P_{+}=(W, \mathbf{0}), P_{-}=\left(E_{B},-\mathbf{q}\right)$, with $E_{B}=\sqrt{M_{B}^{2}+\mathbf{q}^{2}}$. The momentum transfer takes the form $q=(\omega, \mathbf{q})$, where

$$
\omega=\frac{W^{2}-M_{B}^{2}+q^{2}}{2 W}, \quad|\mathbf{q}|=\frac{\sqrt{y_{+} y_{-}}}{2 W} .
$$

The spacelike region, $q^{2} \leq 0$, is characterized by $|\mathbf{q}| \geq|\mathbf{q}|_{0}$ and the timelike region, $\left(W-M_{B}\right)^{2} \geq q^{2}>0$, is characterized by $0 \leq|\mathbf{q}|<|\mathbf{q}|_{0}$, where $|\mathbf{q}|_{0}=\frac{W^{2}-M_{B}^{2}}{2 W}$.

In the calculations, we use the experimental masses $M_{N}=0.939 \mathrm{GeV}, M_{\Lambda}=1.116 \mathrm{GeV}, M_{\Sigma}=1.192 \mathrm{GeV}$ and $M_{\Xi}=1.318 \mathrm{GeV}$, for the octet baryons. As before, $W$ represents the decuplet baryon masses. In the calculations associated with the physical decuplet baryons, we use the physical masses: $M_{\Delta}=1.232 \mathrm{GeV}, M_{\Sigma^{*}}=$ $1.385 \mathrm{GeV}$, and $M_{\Xi^{*}}=1.533 \mathrm{GeV}$.
TABLE III. Coefficients $j_{i}^{S}(i=1,2)$ used to calculate the valence quark contributions for the transition form factors. The label $\gamma^{*} N \rightarrow \Delta$ includes the $\gamma^{*} p \rightarrow \Delta^{+}$and $\gamma^{*} n \rightarrow \Delta^{0}$ transitions ( $n$ is the neutron).

\begin{tabular}{lc}
\hline \hline & $j_{i}^{S}$ \\
\hline$\gamma^{*} N \rightarrow \Delta$ & $\sqrt{2} f_{i-}$ \\
$\gamma^{*} \Lambda \rightarrow \Sigma^{* 0}$ & $\sqrt{\frac{3}{2}} f_{i-}$ \\
$\gamma^{*} \Sigma^{+} \rightarrow \Sigma^{*+}$ & $\frac{\sqrt{2}}{6}\left(f_{i+}+3 f_{i-}+2 f_{i 0}\right)$ \\
$\gamma^{*} \Sigma^{0} \rightarrow \Sigma^{* 0}$ & $\frac{\sqrt{2}}{6}\left(f_{i+}+2 f_{i 0}\right)$ \\
$\gamma^{*} \Sigma^{-} \rightarrow \Sigma^{*-}$ & $\frac{\sqrt{2}}{6}\left(f_{i+}-3 f_{i-}+2 f_{i 0}\right)$ \\
$\gamma^{*} \Xi^{0} \rightarrow \Xi^{* 0}$ & $\frac{\sqrt{2}}{6}\left(f_{i+}+3 f_{i-}+2 f_{i 0}\right)$ \\
$\gamma^{*} \Xi^{-} \rightarrow \Xi^{*-}$ & $\frac{\sqrt{2}}{6}\left(f_{i+}-3 f_{i-}+2 f_{i 0}\right)$ \\
\hline \hline
\end{tabular}

The octet baryon radial wave functions take the form proposed on Refs. $[9,10]$ for the study of the octet baryon electromagnetic form factors

$$
\begin{aligned}
& \psi_{N}(P, k)=\frac{N_{N}}{m_{D}\left(\beta_{1}+\chi_{N}\right)\left(\beta_{2}+\chi_{N}\right)}, \\
& \psi_{\Lambda}(P, k)=\frac{N_{\Lambda}}{m_{D}\left(\beta_{1}+\chi_{\Lambda}\right)\left(\beta_{3}+\chi_{\Lambda}\right)}, \\
& \psi_{\Sigma}(P, k)=\frac{N_{\Sigma}}{m_{D}\left(\beta_{1}+\chi_{\Sigma}\right)\left(\beta_{3}+\chi_{\Sigma}\right)}, \\
& \psi_{\Xi}(P, k)=\frac{N_{\Xi}}{m_{D}\left(\beta_{1}+\chi_{\Xi}\right)\left(\beta_{4}+\chi_{\Xi}\right)},
\end{aligned}
$$

where $N_{B}$ are normalization constants and $\beta_{i}(i=1,2,3,4)$ are square momentum-range parameters in units $M_{B} m_{D}$. The parameters determined in Ref. [9], are $\beta_{1}=0.0532$, $\beta_{2}=0.809, \beta_{2}=0.603$ and $\beta_{2}=0.381$. This parametrization reflects the natural order for the size of the baryon cores $\beta_{2}>\beta_{3}>\beta_{4}$.

As for the decuplet baryon, we use the parametrization from Ref. [77]

$$
\begin{gathered}
\psi_{\Delta}(P, k)=\frac{N_{\Delta}}{m_{D}\left(\alpha_{1}+\chi_{\Delta}\right)^{3}}, \\
\psi_{\Sigma^{*}}(P, k)=\frac{N_{\Sigma^{*}}}{m_{D}\left(\alpha_{1}+\chi_{\Sigma^{*}}\right)^{2}\left(\alpha_{2}+\chi_{\Sigma^{*}}\right)}, \\
\psi_{\Xi^{*}}(P, k)=\frac{N_{\Xi^{*}}}{m_{D}\left(\alpha_{1}+\chi_{\Xi^{*}}\right)\left(\alpha_{2}+\chi_{\Xi^{*}}\right)^{2}},
\end{gathered}
$$

where $N_{B^{\prime}}$ are normalization constants and $\alpha_{i}(i=1,2)$ are square momentum-range parameters in units $M_{B} m_{D}$. In the present case the power associated with the factors in $\alpha_{1}$ and 
$\alpha_{2}$ is related with the number of strange quarks $(0,1$ or 2$)$. The radial wave function of the $\Omega^{-}$, unnecessary for the present study, can be found in Ref. [77]. In the calculations we use the values determined in the study of the decuplet baryon electromagnetic form factors $\alpha_{1}=0.3366$ and $\alpha_{2}=0.1630$ [77].

The normalization constants are determined by the conditions

$$
\begin{aligned}
& \int_{k}\left[\psi_{B}(P, k)\right]^{2}=1, \\
& \int_{k}\left[\psi_{B^{\prime}}(P, k)\right]^{2}=1 .
\end{aligned}
$$

We consider positive values for all normalization constants. The signs of the transition form factors are consequence of these conventions.

The octet baryon $\left(\psi_{B}\right)$ and decuplet baryon $\left(\psi_{B^{\prime}}\right)$ radial wave functions, presented above, ensure that the valence quark contribution to $G_{M}$ defined by Eq. (3.9) is proportional to $1 / Q^{4}$ for very large $Q^{2}$ [72], consistent with estimates from perturbative QCD (pQCD) [92].

The parametrizations of the octet baryon and decuplet baryon radial wave functions were obtained from fits to the lattice QCD simulations of the electromagnetic form factors for pion masses larger than $350 \mathrm{MeV}$ (small meson cloud contributions) $[10,77,93,94]$. The estimates of the valence quark contributions to the octet baryon and decuplet baryon elastic form factors are extrapolated to the physical regime using our extension of the model from the lattice to the physical case. Details of the procedure can be found in Refs. $[9,10,74,77,86]$.

Our estimates for the $\gamma^{*} N \rightarrow \Delta(1232)$ transition form factors compare very well with the lattice QCD simulations with the corresponding pion masses [74]. Our results are also consistent with the bare core estimates from the EBAC model $[30,74]$. The EBAC model is a meson-baryon coupled-channel dynamical model where the mesonbaryon couplings are calibrated by the pion electroproduction data and photo-production data [4,95]. The contributions of the bare core are obtained when we set the meson-baryon coupling to zero [95].

Based on the results for the $\gamma^{*} N \rightarrow \Delta(1232)$ for the lattice QCD regime, where meson cloud effects are negligible, and on the comparison with the EBAC results at the physical point, one can conclude that the calibration of the valence quark degrees of freedom is under control [13]. Our parametrizations of the pion cloud contributions, discussed below, are inferred from the comparison between the extrapolation to the physical limit and the physical data $[3,30,73]$.

A final note about the global normalization of the wave functions is in order. The wave functions associated to the baryon decuplet are normalized properly because the decuplet baryons are described by a model where we neglect the pion cloud contributions. As for the baryon octet, the normalization of the valence quark component is

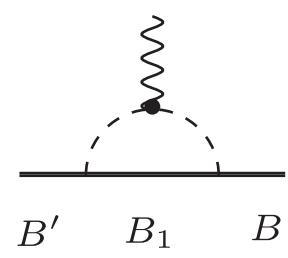

(a)

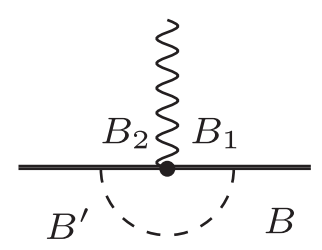

(b)
FIG. 1. Meson cloud contributions for the electromagnetic transition form factors. Between the initial octet $(B)$ and final decuplet $\left(B^{\prime}\right)$ baryon states, there are several possible intermediate baryon states: $B_{1}$ in diagram (a); $B_{1}$ and $B_{2}$ in diagram (b).

modified due to the inclusion of the pion cloud component. We note, however, that this correction only affects $G_{M}^{\mathrm{B}}$ and that, due to the magnitude of the normalization constant and the relative contribution from the valence quark contributions, the normalization effects can be estimated as 3\% at most. One concludes, then, that in a first approximation, we can ignore the normalization correction due to the pion cloud dressing.

\section{Pion cloud contributions}

The pion cloud contribution to the $\gamma^{*} B^{\prime} \rightarrow B$ transition are estimated by the $S U(3)$ extension of our pion cloud model for the $\gamma^{*} N \rightarrow \Delta(1232)$ transition [72-74].

We use, in particular the results of Ref. [13], where the meson cloud contributions of the diagrams of Fig. 1 are determined explicitly in the limit $q^{2}=0$. The calculations of the meson cloud loops are based on the cloudy bag model [96-98]. The explicit calculations use the mesonbaryon couplings for the possible octet baryon and decuplet baryon intermediate states from Fig. 1. The connection with the quark microscopic properties between the covariant spectator quark model and the cloudy bag model is performed matching the Dirac and Pauli couplings. In Ref. [13], in addition to the pion, we considered also the contributions of the kaon and the eta [13]. The eta contributions prove to be very small. More details about the meson and baryon contributions to the processes from Fig. 1 are included in Appendix C.

In the present work, we consider the simplest approximation, taking into account only the pion cloud contributions, and drop the kaon cloud contributions, since the extrapolation of the pion cloud contributions to finite $q^{2}$, based on the results of the $\gamma^{*} N \rightarrow \Delta(1232)$ transition is straightforward.

The generalization of the pion cloud contributions to the timelike region follows the lines of our work for the $\gamma^{*} N \rightarrow$ $\Delta(1232)$ [30]. We represent then

$$
\begin{aligned}
G_{M}^{\pi}\left(q^{2}\right)= & G_{M}^{\pi \mathrm{a}}(0) F_{\pi}\left(q^{2}\right)\left(\frac{\Lambda_{\pi}^{2}}{\Lambda_{\pi}^{2}-q^{2}}\right)^{2} \\
& +G_{M}^{\pi \mathrm{b}}(0) \tilde{G}_{D}^{2}\left(q^{2}\right),
\end{aligned}
$$


where $G_{M}^{\pi \mathrm{a}}(0)$ and $G_{M}^{\pi \mathrm{b}}(0)$ are the pion contributions for the diagrams (a) and (b) in the limit $q^{2}=0$, respectively, $F_{\pi}$ is the pion electromagnetic form factor, $\Lambda_{\pi}^{2}=1.53 \mathrm{GeV}^{2}$, and $\tilde{G}_{D}$ is a generalization of the traditional dipole form factor. The coefficients $G_{M}^{\pi \mathrm{a}}(0)$ and $G_{M}^{\pi \mathrm{b}}(0)$ are presented in Table IV. In Eq. (3.21) we omit the dependence on $W$, since the coefficients $G_{M}^{\pi \mathrm{a}}(0)$ and $G_{M}^{\pi \mathrm{b}}(0)$ are determined in the physical limit $\left(W=M_{B^{\prime}}\right)$.

We use the parametrization [30]

$$
F_{\pi}\left(q^{2}\right)=\frac{\alpha}{\alpha-q^{2}-\frac{1}{\pi} \beta q^{2} \log \frac{q^{2}}{m_{\pi}^{2}}+i \beta q^{2}},
$$

where $\alpha=0.696 \mathrm{GeV}^{2}, \beta=0.178$ and $m_{\pi}$ is the mass of the pion. In the spacelike region $F_{\pi}$ takes the form (analytic continuation)

$$
F_{\pi}\left(q^{2}\right)=\frac{\alpha}{\alpha-q^{2}-\frac{1}{\pi} \beta q^{2} \log \frac{\left(-q^{2}\right)}{m_{\pi}^{2}}} .
$$

Equation (3.22) is derived from an analytic expression which include the structure of the two-pion threshold $[30,51,75]$ for $q^{2} \gg 4 m_{\pi}^{2}$, in order to obtain a simpler parametrization of the $F_{\pi}$ data. Although the two-pion structure is not included explicitly, the error in the approximation is small, since the imaginary component has a small magnitude in the region $0 \leq q^{2} \leq 4 m_{\pi}^{2}$. One derives, then a smoother approximation to the imaginary part of $F_{\pi}$ without significant loss of accuracy. Higher precision parametrizations based on more complex analytic structures and a larger number of parameters can be found in Refs. [99-101].

Following Ref. [30], the function $\tilde{G}_{D}$ is defined as

$$
\tilde{G}_{D}\left(q^{2}\right)=\frac{\Lambda_{D}^{4}}{\left(\Lambda_{D}^{2}-q^{2}\right)^{2}+\Lambda_{D}^{2} \Gamma_{D}^{2}},
$$

where $\Lambda_{D}^{2}=0.9 \mathrm{GeV}^{2}$ and $\Gamma_{D}\left(q^{2}\right)$ is an effective width. The explicit expression for $\Gamma_{D}\left(q^{2}\right)$ is presented in Appendix B.

The parametrization of (3.21) is motivated by the fast suppression of the pion cloud contributions in the spacelike region. This effect is simulated by simple multipole functions, and with the direct photon coupling with the pion in the diagram 1(a). The second term simulates the contributions from the diagram 1(b) and therefore includes the contributions from several intermediate electromagnetic transitions between octet and/or decuplet baryon states (check Appendix C). The multipole powers are chosen using the expected falloff for large $Q^{2}$, estimated by pQCD [92]. Analysis based on pQCD suggests that the valence quark contributions dominate $G_{M}$ and that $G_{M} \propto 1 / Q^{4}$.
TABLE IV. Coefficients of the pion cloud contributions. In the last column, we include the bare contribution at $q^{2}=0$.

\begin{tabular}{lrrrr}
\hline \hline & $G_{M}^{\pi \mathrm{a}}(0)$ & $G_{M}^{\pi \mathrm{b}}(0)$ & $G_{M}^{\pi}(0)$ & $G_{M}^{\mathrm{B}}\left(0, M_{B^{\prime}}\right)$ \\
\hline$\gamma^{*} N \rightarrow \Delta$ & 0.713 & 0.610 & 1.323 & 1.633 \\
$\gamma^{*} \Lambda \rightarrow \Sigma^{* 0}$ & 0.669 & 0.358 & 1.027 & 1.683 \\
& & & & \\
$\gamma^{*} \Sigma^{+} \rightarrow \Sigma^{*+}$ & 0.149 & 0.513 & 0.663 & 2.094 \\
$\gamma^{*} \Sigma^{0} \rightarrow \Sigma^{* 0}$ & 0.000 & 0.270 & 0.270 & 0.969 \\
$\gamma^{*} \Sigma^{-} \rightarrow \Sigma^{*-}$ & -0.149 & 0.026 & -0.124 & -0.156 \\
$\gamma^{*} \Xi^{0} \rightarrow \Xi^{* 0}$ & 0.222 & 0.086 & 0.308 & 2.191 \\
$\gamma^{*} \Xi^{-} \rightarrow \Xi^{*-}$ & -0.222 & 0.084 & -0.138 & -0.168 \\
\hline \hline
\end{tabular}

Extending the analysis for the meson cloud effects, interpreted as the contributions of meson-baryon systems, one concludes that those contributions ${ }^{2}$ are ruled at very large $Q^{2}$ by $G_{M} \propto 1 / Q^{8}$. The second term of (3.21) falls off with $1 / Q^{8}$. The first term of (3.21) falls of with $1 /\left(Q^{6} \log Q^{2}\right)$, still close to the expected rule.

The extension of the model with the inclusion of the kaon cloud will require the generalization of the two terms from Eq. (3.21) to the case of the kaon. This nontrivial generalization is planed for a future work.

In the last column of Table IV, we include for convenience the bare contribution $G_{M}^{\mathrm{B}}\left(0, M_{B^{\prime}}\right)$ to the magnetic form factor at $q^{2}=0$. The relative magnitude of the pion cloud contribution at $q^{2}=0$ can then be estimated by $G_{M}^{\pi}(0) /\left(G_{M}^{\mathrm{B}}\left(0, M_{B^{\prime}}\right)+G_{M}^{\pi}(0)\right)$.

\section{Transition form factors}

We now discuss the results for the transition form factor associated to Eqs. (3.8), (3.9) and (3.21). Our transition form factors are real functions (by construction) in the spacelike region, and became complex only in the timelike region $\left(q^{2}>0\right)$. We present the results for $\left|G_{M}\right|$, because only the magnitude of $G_{M}$ is relevant for the radiative and Dalitz decays. The sign of $G_{M}$ in the spacelike region is the consequence of the our convention to the flavor states presented in Tables I and II.

The numerical results for $\left|G_{M}\right|$, for several values of $W$ near the physical mass $M_{B^{\prime}}$ are presented in Fig. 2 by the thick lines. For the $\Sigma^{*}$ decays the we choose a range of variation based on the $\Sigma^{*}$ total decay width, and on the

\footnotetext{
${ }^{2}$ Using $\mathrm{pQCD}$ one can show that the leading order form factor with $n$ active constituents behaves for large $Q^{2}$ like $1 / Q^{2(n-2)}$. For a system of three quark, one obtains the a falloff with $1 / Q^{4}$. For a system of three quarks and a quark-antiquark pair (5 constituents), resembling a baryon-meson system, one expect then a falloff with $1 / Q^{8}$. Meson cloud contributions are then characterized by an extra suppression of $1 / Q^{4}$ for large $Q^{2}$.
} 

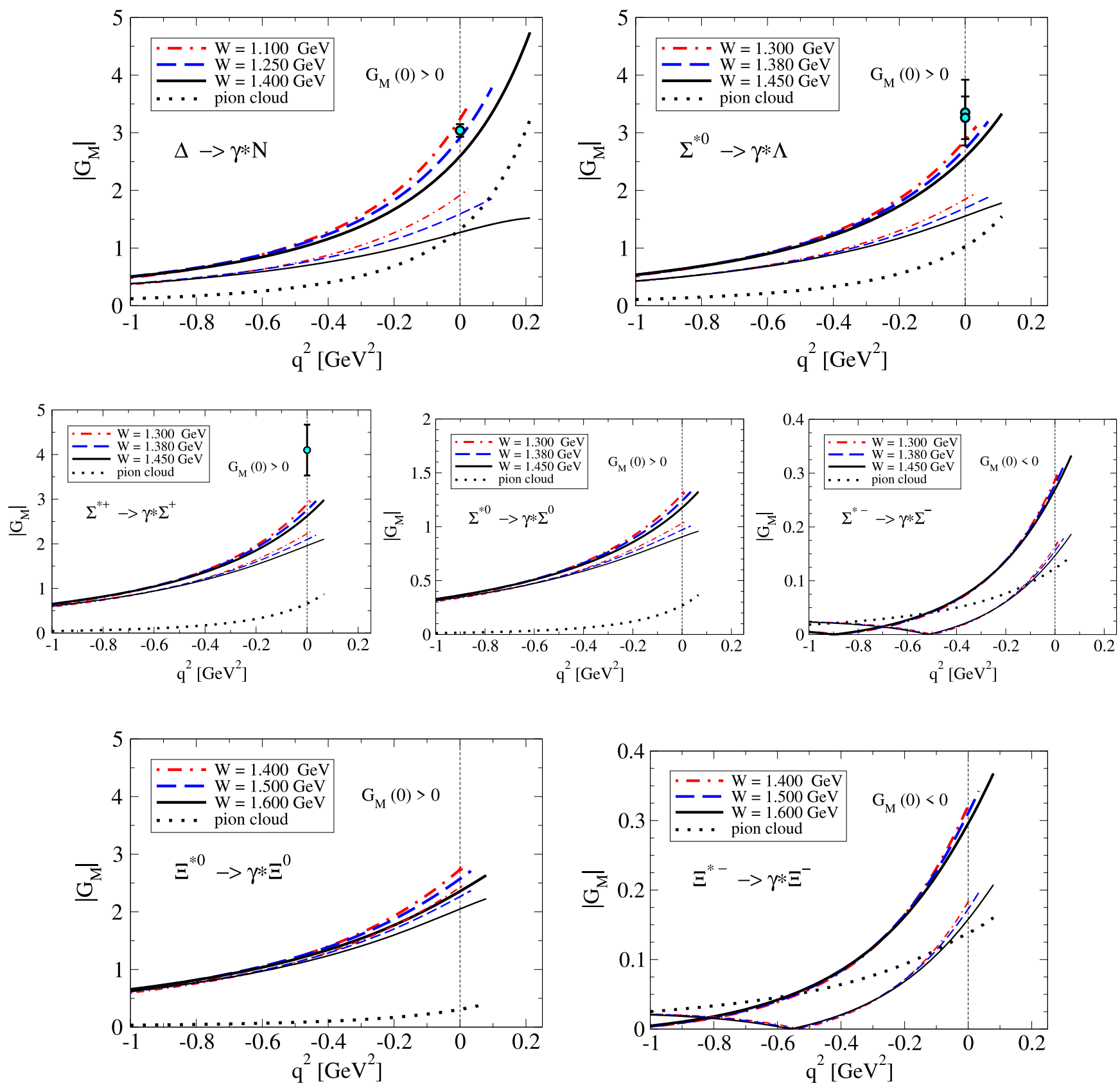

FIG. 2. Magnitude of transition form factor $G_{M}$. The thick lines represent the total (valence plus pion cloud) and the thin lines represent the valence quark contribution. The dotted line represent the pion cloud contributions (follow the discussion in the main text). The data are from Table V.

range of the HADES simulations [42]. For the $\Xi^{*}$ decays, since the decay width is very small (about $10 \mathrm{MeV}$ ), we consider a wider range for a better visualization of the dependence on $q^{2}$.

In addition to $\left|G_{M}\right|$, we include also the result of the valence quark contribution $\left|G_{M}^{\mathrm{B}}\right|$ (thin lines) and the absolute values of the pion cloud contribution (dotted line), according to Eq. (3.21). The line associated to the pion cloud corresponds, in fact, to the estimate associated with the largest value of $W$. The remaining cases have the same shape, except that the estimates are limited to $q^{2} \leq\left(W-M_{B}\right)^{2}$.
The data included in the graph represent the magnitude of the experimental magnetic form factors for $q^{2}=0$, estimated from the radiative decay width data. The $G_{M}(0)$ data is discussed in more detail in the next section. The experimental values for $\left|G_{M}(0)\right|$ are important to infer the accuracy of the constant form factor model. The model associated with the constant form factor corresponds to a horizontal line with the magnitude of the experimental value for $\left|G_{M}(0)\right|$.

One can notice that the model estimates for the $\Delta \rightarrow \gamma^{*} N$ and $\Sigma^{* 0} \rightarrow \gamma^{*} \Lambda$ decays have a magnitude comparable with 
TABLE V. Results for $G_{M}(0)$ corresponding to the $B^{\prime} \rightarrow \gamma B$ decays. The values for $\left|G_{M}(0)\right|_{\text {exp }}$ are estimated using the experimental values of $\Gamma_{B^{\prime} \rightarrow \gamma B} .\left.G_{M}(0)\right|_{\pi}$ is the estimate when we omit the kaon cloud contributions (only pion cloud).

\begin{tabular}{|c|c|c|c|c|c|}
\hline & $G_{M}(0)$ & $\left.G_{M}(0)\right|_{\pi}$ & $\left|G_{M}(0)\right|_{\exp }$ & $\Gamma(\mathrm{keV})$ & $\Gamma_{\exp }(\mathrm{keV})$ \\
\hline$\Delta \rightarrow \gamma N$ & 3.02 & 2.96 & $3.04 \pm 0.11[102]$ & 648 & $660 \pm 47[102]$ \\
\hline$\Sigma^{* 0} \rightarrow \gamma \Lambda$ & 3.08 & 2.71 & $\begin{array}{c}3.35 \pm 0.57[102] \\
3.26 \pm 0.37[59]\end{array}$ & 399 & $\begin{array}{c}470 \pm 160[102] \\
445 \pm 102[59]\end{array}$ \\
\hline$\Sigma^{*+} \rightarrow \gamma \Sigma^{+}$ & 3.22 & 2.76 & $4.10 \pm 0.57[103]$ & 154 & $250 \pm 70[103]$ \\
\hline$\Sigma^{* 0} \rightarrow \gamma \Sigma^{0}$ & 1.46 & 1.24 & $<11[104]$ & 32 & $<1750[104]$ \\
\hline$\Sigma^{*-} \rightarrow \gamma \Sigma^{-}$ & -0.31 & -0.28 & $<0.8[105]$ & 1.4 & $<9.5[105]$ \\
\hline$\Xi^{* 0} \rightarrow \gamma \Xi^{0}$ & 3.29 & 2.50 & & 182 & \\
\hline$\Xi^{*-} \rightarrow \gamma \Xi^{-}$ & -0.38 & -0.31 & $<4.2[106]$ & 2.4 & $<366$ [106] \\
\hline
\end{tabular}

the data. In the case of the $\Sigma^{*+} \rightarrow \gamma^{*} \Sigma^{+}$decay the model underestimate clearly the data. This underestimation is in part the consequence of neglecting the kaon cloud contributions. When those effects are taken into account one obtain $G_{M}(0)=3.22$, only 1.5 standard deviations from below the data [13].

In Fig. 2, one can observe the dependence of the transition form factors on the variable $W$. In general, for a fixed value of $q^{2}$ the magnitude of $G_{M}$ decreases with $W$, as a consequence of our analytic expressions for $G_{M}^{\mathrm{B}}$. This $W$-dependence was tested in our calculations in the lattice QCD regime, where the masses of the baryons and mesons are larger that the physical ones $[3,74,86]$. The $W$-dependence of our results is an important characteristic of our formalism, which has an impact on the calculation of the radiative and the Dalitz decay widths in terms of $W$, presented in the Sec. IV C.

In this aspect the present model is distinct of other models, like the constant form factor model and some VMD models [50]. The Iachello-Wan model [29,51] includes only a weak $W$-dependence on the transition form factors.

In the graphs, the spacelike results for $G_{M}$ are equivalent to the results presented in the graph for $\left|G_{M}\right|$, in most cases, since $G_{M}(0)>0$. The exceptions are the $\Sigma^{*-} \rightarrow \gamma^{*} \Sigma^{-}$and $\Xi^{*-} \rightarrow \gamma^{*} \Xi^{-}$decays, where $G_{M}(0)<0$, according to the estimates from Ref. [13]. Our numerical values for $G_{M}(0)$ are presented in the next section (see Table V).

The results for the $\Delta \rightarrow \gamma^{*} N$ form factors are almost identical to the results from Ref. [30], except that in the previous work we use the approximation $G_{M}^{\pi \mathrm{a}}(0)=$ $G_{M}^{\pi \mathrm{b}}(0)=\frac{1}{2} G_{M}^{\pi}(0)$ (pion cloud contributions equally divided between the two pion cloud processes from Fig. 1). The results for the $\Delta \rightarrow \gamma^{*} N$ form factors are interesting because there is a deeper penetration in the timelike region due the large values of the upper limit $\left(W-M_{B}\right)^{2}$, where $B$ is the nucleon. For larger values of $W$ one can notice that the valence quark contribution line became more flat.
In that region, one can also observe the enhancement of $\left|G_{M}\right|$ for large $q^{2}$, a direct consequence of the pion cloud contribution regulated by Eqs. (3.21) and (3.22), characterized by the peak of $F_{\pi}\left(q^{2}\right)$ near $q^{2} \approx m_{\rho}^{2} \simeq 0.6 \mathrm{GeV}^{2}$.

The first detailed study of the $\Delta(1232)$ Dalitz decay at HADES suggests that the constant form factor model is insufficient to describe the data and that the signature of the form factor dependence on $q^{2}$ is present in the data [15].

The present calculations also suggest that the constant form factor model is not a good approximation for the $\Sigma^{* 0} \rightarrow \gamma^{*} \Lambda, \Sigma^{*-} \rightarrow \gamma^{*} \Sigma^{-}$and $\Xi^{*-} \rightarrow \gamma^{*} \Xi^{-}$decays, since in those cases $\left|G_{M}\right|$ is significantly enhanced near the pseudothreshold. Those enhancements can be the consequence of the bare contribution $\left(\Sigma^{*-} \rightarrow \gamma^{*} \Sigma^{-}\right.$and $\left.\Xi^{*-} \rightarrow \gamma^{*} \Xi^{-}\right)$or the pion cloud contribution $\left(\Sigma^{* 0} \rightarrow \gamma^{*} \Lambda\right)$.

The results for the $\Sigma^{*+} \rightarrow \gamma^{*} \Sigma^{+}, \Sigma^{* 0} \rightarrow \gamma^{*} \Sigma^{0}$ and $\Xi^{* 0} \rightarrow$ $\gamma^{*} \Xi^{0}$ transitions indicate that the relative pion cloud contributions are smaller than in the other transitions.

From the graphs for $\left|G_{M}\right|$, we also conclude that there are different classes of magnitudes: $\Delta \rightarrow \gamma^{*} N$ and $\Sigma^{* 0} \rightarrow \gamma^{*} \Lambda$; $\Sigma^{*+} \rightarrow \gamma^{*} \Sigma^{+}$and $\Xi^{* 0} \rightarrow \gamma^{*} \Xi^{0}$ (large magnitude); $\Sigma^{* 0} \rightarrow$ $\gamma^{*} \Sigma^{0}$ (moderate magnitude); $\Sigma^{*-} \rightarrow \gamma^{*} \Sigma^{-}$and $\Xi^{*-} \rightarrow \gamma^{*} \Xi^{-}$ (small magnitude) [12]. The impact of these magnitudes on the Dalitz decay widths is discussed in Sec. IV B.

In the graphs for the $\Sigma^{*-} \rightarrow \gamma^{*} \Sigma^{-}$and $\Xi^{*-} \rightarrow \gamma^{*} \Xi^{-}$ transitions, one can notice nodes in both $\left|G_{M}\right|$ and $\left|G_{M}^{\mathrm{B}}\right|$ in the spacelike region. Those nodes are a consequence of zeros of $G_{M}$ due to a sign change. Since both form factors are negative near $q^{2}=0$, the nodes indicate the point where the functions became negative. The zero crossings are the consequence of the sign change of the valence quark contributions, according to our $S U(3)$ parametrization of the quark form factors (see Table III). Similar results were also obtained in a previous study based on the covariant spectator quark model [12], with a not so general description of the pion cloud contributions.

The $\Sigma^{*-} \rightarrow \gamma^{*} \Sigma^{-}$and $\Xi^{*-} \rightarrow \gamma^{*} \Xi^{-}$transitions are the transitions with smaller valence quark contributions. This 
result is also a consequence of our approximated $S U(3)$ symmetry. In the exact $S U(3)$ limit the form factors $f_{i+}$, $f_{i-}$ and $f_{i s}$ are undistinguished and the valence quark contribution vanishes because $j_{i}^{S} \equiv 0$, according to Table III. The small but nonzero contributions to $G_{M}^{\mathrm{B}}$ are then the consequences of a small $S U(3)$ symmetry breaking.

\section{E. Comparison with the literature}

Our estimates can be compared directly with other estimates based on valence quark degrees of freedom.

Calculations based on nonrelativistic and relativistic quark models $[47,56-58,60]$ underestimate in general $G_{M}$ near $Q^{2}=0$, which may be interpreted as a consequence of the absence of meson cloud effects. Also lattice QCD simulations underestimate $G_{M}(0)$ [63]. In Ref. [12], we compare explicitly our upper limit for the valence quark contribution for $G_{M}(0)$, defined by Eq. (3.9) with $\mathcal{I}\left(0, M_{B^{\prime}}\right)=1$, with the lattice results from Ref. [63]. We conclude that the two estimates are very close, within the lattice QCD uncertainties.

We now compare our estimates of the valence quark contributions with estimates based on the DysonSchwinger framework from Ref. [62], also based on the valence quark degrees of freedom. Our results for $G_{M}^{B}$ compare well with the estimates from Ref. [62] above $1 \mathrm{GeV}^{2}$, for transitions with larger magnitude for $\left|G_{M}\right|$, suggesting that the two methods have similar predictions for the large- $Q^{2}$ region. For the $\Sigma^{-*}$ and $\Xi^{-*}$ decays, both formalisms predict small but different magnitudes. Recall that those transitions are more sensitive to the mechanisms of $S U(3)$ symmetry breaking. Both formulations predict that the quark core contributions vanish in some point between 0 and $1 \mathrm{GeV}^{2}$. Below $Q^{2}=1 \mathrm{GeV}^{2}$, the comparison is more delicate, because the Dyson-Schwinger estimates are presented as an interval of variation. From the results for $Q^{2}=0.1$ and $0.2 \mathrm{GeV}^{2}$, one can conclude that we overestimate the results from Ref. [62] in about $30 \%-50 \%$.

The transition form factors have also been calculated with a $S U(3)$ chiral quark-soliton model [68], taking into account some pion production from the nucleon. The model explains well the $\gamma^{*} N \rightarrow \Delta(1232)$ lattice QCD data for $G_{M}$ for the corresponding pion mass. The model calibrated by $Q^{2} \simeq 0$ data describe well the low- $Q^{2}$ data but falls off slower that the experimental data. The estimates of the reaming transition form factors compare well with our estimates of the bare contribution to $G_{M}(0)$ (see Table IV), but differ in sign. The unnormalized estimate of $G_{M}$ for the $\gamma^{*} N \rightarrow \Delta(1232)$ transition is also similar to our estimate for $G_{M}^{\mathrm{B}}(0)$. Their form factors have a slower falloff with $Q^{2}$ when compared with our estimates.

When we restrict the analysis to $Q^{2}=0$ there are several frameworks which provide estimates for $\left|G_{M}(0)\right|$ closer to the available data. There are calculations based on chiral perturbation theory $[46,69,70]$ and the large $N_{c}$ limit [71]. Those estimates are restricted in the range of $Q^{2}$, and rely on the determination of low-energy constants. Also calculations based on QCD sum rules predict large contributions to $\left|G_{M}(0)\right|$ in comparison with our estimates $[64,65]$. One notices, however, that those the comparison between quark models and QCD sum rules have to be performed with care, since the normalization in QCD sum rules is based on distribution amplitudes defined for large $Q^{2}$, in contrast with quark models, where the normalization is defined at $Q^{2}=0$.

In the next section, we study the impact of our model for the transition form factors on the radiative and Dalitz decay widths.

\section{RADIATIVE AND DALITZ DECAY WIDTHS}

We present here our estimates for the $B^{\prime}$ radiative and Dalitz decay widths. We start with the radiative decays at the pole: $\Gamma_{\gamma B}\left(M_{B^{\prime}}\right)$. Later on, we discuss the functions $\frac{d}{d q} \Gamma_{e^{+} e^{-} B}(q, W), \Gamma_{e^{+} e^{-} B}(W)$ and $\Gamma_{\gamma B}(W)$.

\section{A. Electromagnetic decay widths}

Using the dominance of the magnetic dipole form factor, we can write $[9,10,50]$

$$
\Gamma_{\gamma B}=\frac{\alpha}{16} \frac{\left(M_{B^{\prime}}^{2}-M_{B}^{2}\right)^{3}}{M_{B^{\prime}}^{3} M_{B}^{2}}\left|G_{M}(0)\right|^{2} .
$$

In Table V, we present the model estimates for $G_{M}(0)$ and $\Gamma \equiv \Gamma_{\gamma B}$ in the second and fifth columns, and compare those estimates with the experimental data [6,102-106], in the fourth and sixth columns. $\left|G_{M}(0)\right|_{\exp }$ is determined from $\Gamma_{\exp }$ using Eq. (4.1). The numerical results were calculated in Ref. [13]. For the decays for which there are no data, we include the experimental estimate of the upper limit when available.

The estimate of the third column, $\left.G_{M}(0)\right|_{\pi}$, correspond to the calculation which exclude the kaon cloud contribution (only pion cloud), as in Sec. III D.

As discussed in the previous section, our estimate of $G_{M}(0)$, given by $\left.G_{M}(0)\right|_{\pi}$, is consistent with the data for the $\Delta \rightarrow \gamma N$ and $\Sigma^{*+} \rightarrow \gamma \Lambda$ decays, and underestimates the result for the $\Sigma^{*+} \rightarrow \gamma \Sigma^{+}$decay. Calculations based on chiral perturbation theory [46,69], large $N_{c}$ limit [71] and QCD sum rules [65], compare well with the available data. A detailed comparison between model estimates and experimental data can be found in Refs. [12,13].

On Table V, one can notice that the experimental limit for the $\Sigma^{*-}$ decay is close to our model estimate. One can conclude then that there is some hope that this decay width can be measured in a near future.

\section{B. Dalitz decay rates}

The results for the Dalitz decay rates are presented in Fig. 3, for all the decuplet baryon decays, for several values of $W$. We include the labels $B^{\prime} \rightarrow e^{+} e^{-} B$ in order to 

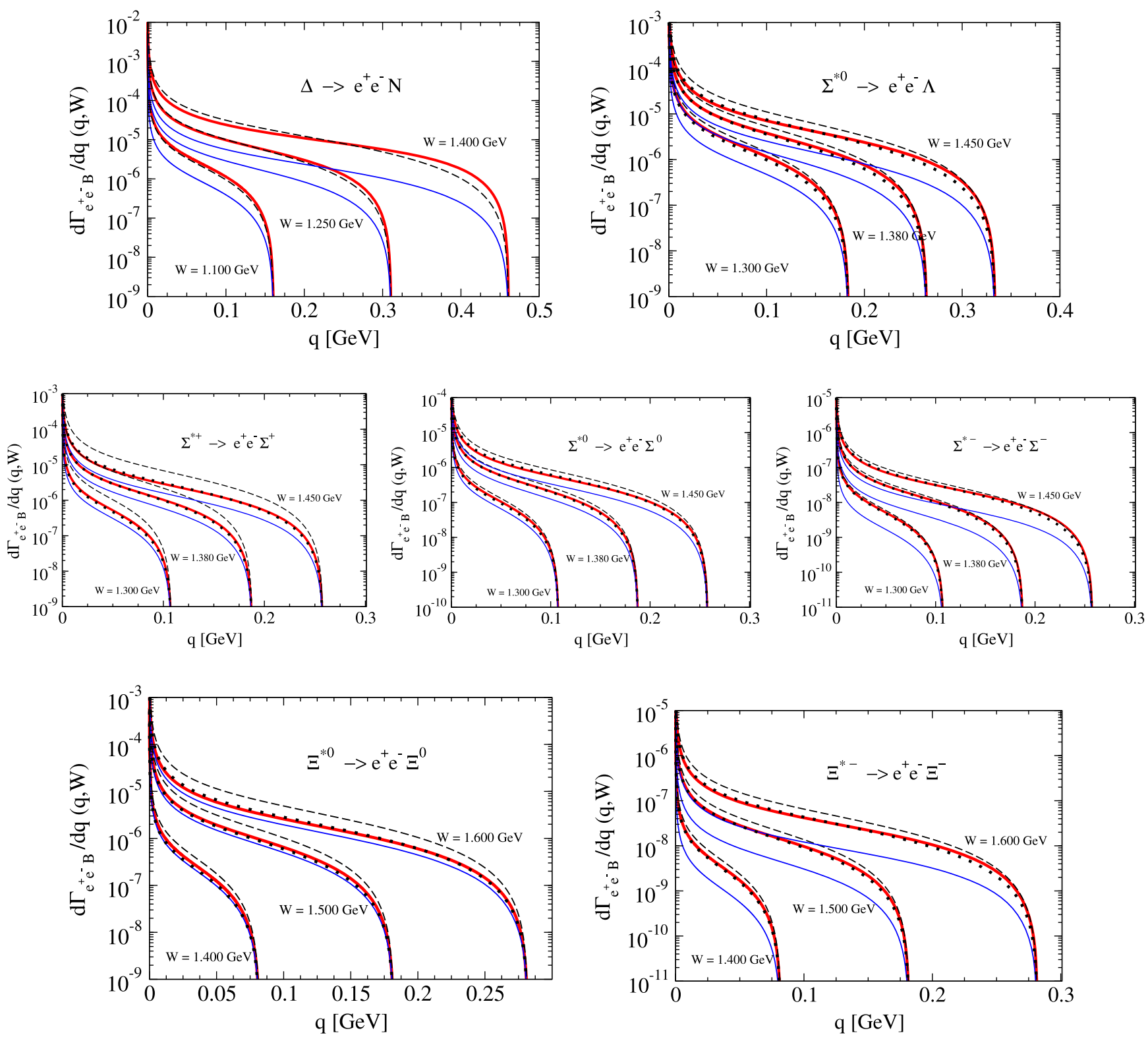

FIG. 3. Dalitz decay rates $\frac{d}{d q} \Gamma_{e^{+} e^{-} B}$ for different values of $W$. Note a difference of scales. The thick solid lines represent our final estimation (bare plus pion cloud). The thin solid lines represent the bare quark approximations. The results of the constant form factor model $\left[G_{M}\left(q^{2}\right) \rightarrow G_{M}(0)\right]$ are indicated by the dashed lines. The dotted lines represent the estimate of the constant form factor model when we exclude the kaon cloud $\left(\left.G_{M}\left(q^{2}\right) \rightarrow G_{M}(0)\right|_{\pi}\right)$.

identify the decaying decuplet baryon. Recall that $\Sigma^{0}(1385)$ decay on $\Lambda(1116)$ and on $\Sigma^{0}(1193)$.

The thick solid lines indicate the final result: the combination of valence quark and pion cloud contributions. The thin lines indicate the valence quark contributions (when we drop the pion cloud contributions).

The dashed lines indicate the result of the constant form factor model, obtained when we consider: $G_{M} \equiv G_{M}(0)$, also known as QED estimate. To represent the QED estimate, we consider the following convention:

(i) In cases where experimental data exist $(\Delta \rightarrow \gamma N$, $\Sigma^{* 0} \rightarrow \gamma \Lambda$ and $\Sigma^{*+} \rightarrow \gamma \Sigma^{+}$), we use the magnetic form factor determined by the electromagnetic decay width (see Table V). For the $\Sigma^{* 0} \rightarrow \gamma \Lambda$ transition we approximate the result by the central value $\left(\left|G_{M}(0)\right|_{\exp } \simeq 3.3\right)$.

(ii) In the remaining cases, we use our best estimate given by the results from Table $\mathrm{V}$, corresponding to the value of $G_{M}(0)$ which include the pion and kaon clouds (second column).

For the discussion of the $q^{2}$-dependence of our model, we include also the model estimate of the Dalitz decay when we replace $G_{M}\left(q^{2}\right)$, by $\left.G_{M}(0)\right|_{\pi}$. The results are represented by the dotted lines. In the case of the 
$\Delta \rightarrow e^{+} e^{-} N$ decay we omit this estimate because it overlaps the estimate of the constant form factor model (dashed line).

The importance of the pion cloud contributions is clearly shown in Fig. 3, from the difference between the thick (total) and thin (bare) solid lines. This difference of magnitude is a consequence of the relative magnitude of the corresponding estimates for the transition form factors. We recall that based on the estimates from Ref. [13], also presented in Table IV, the valence quark contributions to the transition form factors are about $55 \%-70 \%$ of the total. One concludes, then, that when the pion cloud contribution are about $50 \%$ of the total, the bare estimates for $\frac{d}{d q} \Gamma_{e^{+} e^{-} B}$ are about $1 / 4$ of the total, since the decay widths are proportional to $\left|G_{M}\right|^{2}$. This rough estimate is valid for most decays. The main exceptions are the $\Sigma^{*+} \rightarrow e^{+} e^{-} \Sigma^{+}, \Sigma^{* 0} \rightarrow e^{+} e^{-} \Sigma^{0}$ and $\Xi^{* 0} \rightarrow e^{+} e^{-} \Xi^{0}$ decays, where the relative contribution of the core is larger (smaller pion cloud contributions).

The magnitudes of the different decays can be clearly observed in the scale of the Dalitz decay widths: large magnitudes for $\Delta \rightarrow e^{+} e^{-} N, \quad \Sigma^{* 0} \rightarrow e^{+} e^{-} \Lambda, \quad \Sigma^{*+} \rightarrow$ $e^{+} e^{-} \Sigma^{+}$and $\Xi^{* 0} \rightarrow e^{+} e^{-} \Xi^{0}$ (scale $10^{-3}$ ); intermediate magnitude for $\Sigma^{* 0} \rightarrow e^{+} e^{-} \Sigma^{0}$ (scale $10^{-4}$ ); small magnitudes for $\Sigma^{*-} \rightarrow e^{+} e^{-} \Sigma^{-}$and $\Xi^{*-} \rightarrow e^{+} e^{-} \Xi^{-}$(scale $10^{-5}$ ) [13]. Those magnitudes are the consequence of the magnitudes of the magnetic form factors discussed in Sec. III D.

Concerning the comparison with the constant form factor model (dashed lines), one can conclude that the results are very close for the $\Delta \rightarrow e^{+} e^{-} N$ decay, for small values of $W$. This happens because our model is compatible with the experimental value for $\left|G_{M}(0)\right|$, as discussed earlier. In the remaining cases, our result underestimates the constant form factor model. This underestimation is mainly a consequence of the noninclusion of the kaon cloud contribution in our $q^{2}$ dependent estimates, in contrast with the constant form factor model. This underestimation was discussed in detail in Sec. III D for the $\Sigma^{*+} \rightarrow \gamma^{*} \Sigma^{+}$form factor.

The impact of the form factor dependence on $q^{2}$ can be inferred from the comparison between the exact estimate (thick solid line) and the dotted line. As anticipated in Sec. III D, the $q^{2}$-dependence is more relevant for the $\Sigma^{* 0} \rightarrow e^{+} e^{-} \Lambda, \Sigma^{*-} \rightarrow e^{+} e^{-} \Sigma^{-}$and $\Xi^{*-} \rightarrow e^{+} e^{-} \Xi^{-}$decays. The dominance of the exact result over the dotted line is clearly observed for large $q^{2}$, particularly for large values of $W$.

In Fig. 4, we compare the magnitudes of the $\Sigma^{*}$ and $\Xi^{*}$ Dalitz decay rates at respective the mass poles. Note the similarity between the results for $\Sigma^{*+} / \Xi^{* 0}$ decays, as well as $\Sigma^{*-} / \Xi^{*-}$ decays in the region of $q^{2}$ where they can be compared. These similarities are the consequence of the $S U$ (3) symmetry structure of the covariant spectator quark model, combined with similar relative pion cloud contributions for the decays under discussion. The relations between

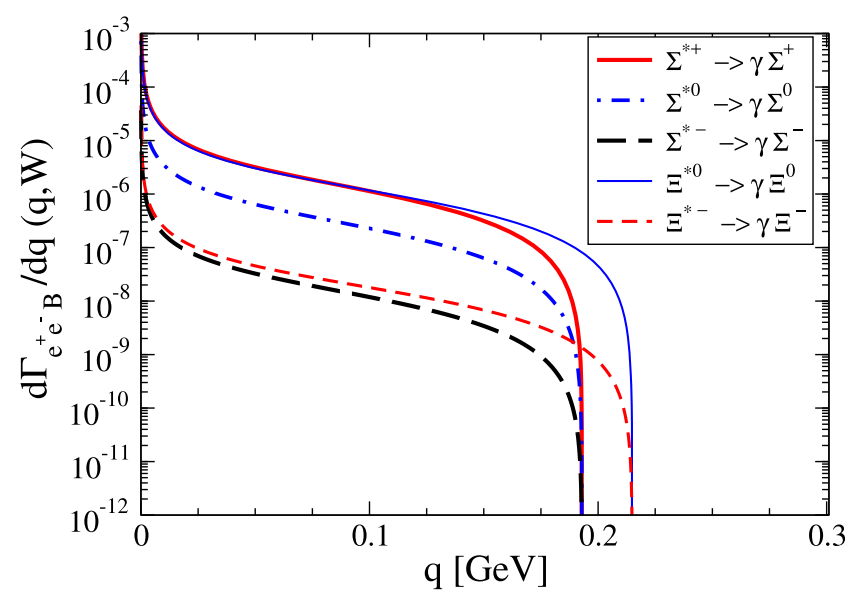

FIG. 4. Comparison between the $\Sigma^{*}$ and $\Xi^{*}$ Dalitz decay rates at the physical point $\left(W \simeq 1.385 \mathrm{GeV}\right.$ for $\Sigma^{*}$ and $W \simeq 1.533 \mathrm{GeV}$ for $\left.\Xi^{*}\right)$.

the valence quark contributions, $G_{M}^{\mathrm{B}}$, given by Eq. (3.9) are explained by their dependence on the functions $j_{i}^{S}$, which, according to Table III are identical in the cases $\Sigma^{*+} / \Xi^{* 0}$ and $\Sigma^{*-} / \Xi^{*-}$.

The similarities between the $\Sigma^{*-}$ and $\Xi^{*-}$ decays are also explained by the $U$-spin symmetry $[13,59]$, which is valid to the valence quark component of the transition form factors. The $U$-spin symmetry, states that the decay transitions are similar when we replace a $d$-quark by a $s$-quark in the initial and final states [59]. The symmetry predicts also similar magnitudes for the $\Delta \rightarrow \gamma N$ and the $\Sigma^{* 0} \rightarrow \gamma \Lambda$ Dalitz decay rates $[12,13]$. This property, however, is not valid in the context of our model due to the difference of magnitudes of the pion cloud contributions (larger in the first case).

Our model is compatible with the $U$-spin symmetry, because it is based on an approximate $S U(3)$ flavor symmetry. In the present case, the symmetry implies that the quark form factors associated with the $u$ quark (combination of isovector and isoscalar components) and the $s$ quark are similar at low $q^{2}$. We recall, however, that the $U$-spin symmetry is valid only for the valence quark component of the transition. The covariant spectator quark model estimates provide then a more consistent description of the radiative and Dalitz decays.

\section{Decay widths in terms of the invariant mass}

The results for the radiative $\left(B^{\prime} \rightarrow \gamma B\right)$ and Dalitz $\left(B^{\prime} \rightarrow e^{+} e^{-} B\right)$ decay widths in terms of $W$ are presented in Fig. 5, for all the decuplet baryon decays. The thick lines represent our estimates. The thin lines represent the estimates of the constant form factor model. We include also the data for $\Gamma_{\gamma B}$ at the physical mass in the cases: $\Delta \rightarrow \gamma N, \quad \Sigma^{* 0} \rightarrow \gamma \Lambda$ and $\Sigma^{*+} \rightarrow \gamma \Sigma^{+}$, according to the results from Table V. 

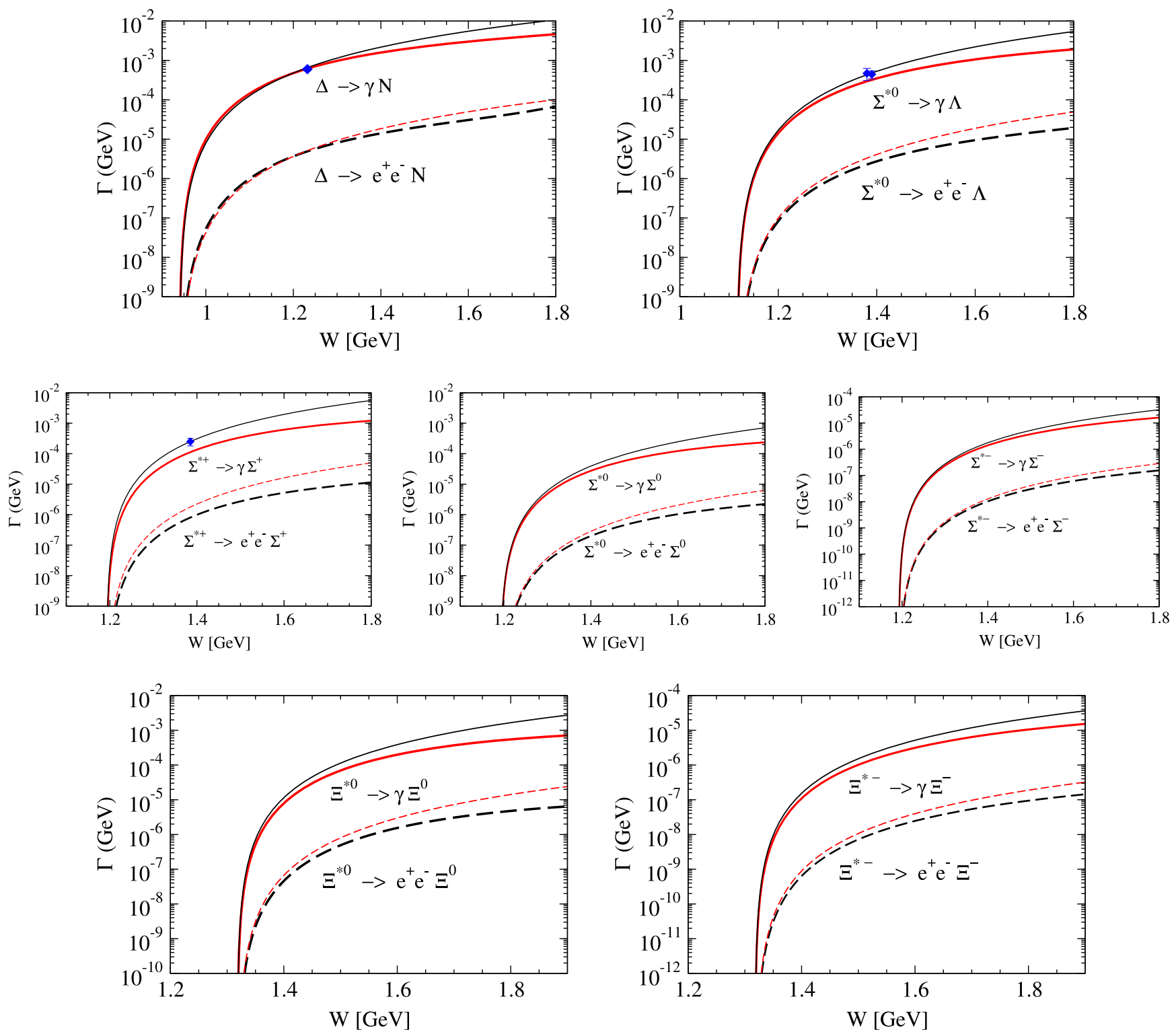

FIG. 5. Electromagnetic and Dalitz decay widths for all the decuplet decays in terms of $W$. The thick lines represent our model. The thin lines represent the constant form factor model. Data from Table V.

In the cases $\Sigma^{* 0} \rightarrow \gamma \Lambda$ and $\Sigma^{*+} \rightarrow \gamma \Sigma^{+}$, one can notice some underestimation of the data. This result is in part the consequence of including only the contribution of the pion cloud. The inclusion of the kaon cloud approaches the model estimate to the data, as can be inferred also from Table $\mathrm{V}$ (compare the second and the third columns).

We choose to not include the kaon cloud contributions on the radiative decays, because our extension for finite $q^{2}$ is justified, at the moment, only for the pion cloud contribution. In general, the kaon cloud contributions are at most $20 \%$ of the pion cloud contributions (Table V), except for $\Xi^{* 0}$, where the effect of the kaon is about $25 \%$ of the pion cloud.
Concerning the comparison with the results of the QED model, there are two points to debate. First, when we use a constant value for $\left|G_{M}\right|$, the results for $\Gamma_{\gamma B}(W)$ are close to the model estimates for $\Gamma_{\gamma B}(W)$ for small values of $W$, and start to overestimate the model above a certain value of $W$. Second, the overestimation of QED model, for large values of $W$ is expected due our model underestimation for $\left|G_{M}\right|$. More definite conclusions can be drawn only when the unknown decay widths are determined experimentally.

Regarding the inclusion of $q^{2}$-dependent kaon cloud contributions, our expectation is that the slope associated with the kaon cloud contributions is larger than the slope associated with the pion cloud contributions near $q^{2}=0$, since the kaon cloud effects are more suppressed than the 
TABLE VI. Decuplet baryon Dalitz decay widths. The HADES result for the $\Delta(1232)$ Dalitz decay is $4.90 \pm 0.83 \mathrm{keV}$ [15].

\begin{tabular}{lc}
\hline \hline Decay & $\Gamma_{e^{+} e^{-} B}(\mathrm{keV})$ \\
\hline$\Delta \rightarrow e^{+} e^{-} N$ & 4.9 \\
$\Sigma^{* 0} \rightarrow e^{+} e^{-} \Lambda$ & 2.4 \\
& \\
$\Sigma^{*+} \rightarrow e^{+} e^{-} \Sigma^{+}$ & 0.81 \\
$\Sigma^{* 0} \rightarrow e^{+} e^{-} \Sigma^{0}$ & 0.16 \\
$\Sigma^{*-} \rightarrow e^{+} e^{-} \Sigma^{-}$ & $0.83 \times 10^{-3}$ \\
$\Xi^{* 0} \rightarrow e^{+} e^{-} \Xi^{0}$ & 0.76 \\
$\Xi^{*-} \rightarrow e^{+} e^{-} \Xi^{-}$ & $1.2 \times 10^{-3}$ \\
\hline \hline
\end{tabular}

pion cloud effects in the spacelike region. ${ }^{3}$ The consequence of this trend is that the transition form factors are expected to be enhanced in the timelike region with the inclusion of the kaon cloud contributions. We recall, however, that the kaon cloud contributions near $q^{2}=0$ are at most about $20 \%$ of the pion cloud contributions. There is then the possibility that the kaon cloud effects may not be very relevant in the region $0<q^{2} \leq\left(W-M_{B}\right)^{2}$. Only more detailed calculations can determine how important may be the enhancement of the transition form factors due to the kaon cloud effects, in the timelike region.

Our estimates for the $B^{\prime} \rightarrow e^{+} e^{-} B$ Dalitz decays underestimate, in general, the QED model. This tendency is a consequence of the results obtained for the Dalitz decay rates (Fig. 3), where the QED model overestimates, in general, the covariant spectator quark model. The exception is the $\Delta \rightarrow e^{+} e^{-} N$ decay, where our model and the QED estimates are close.

It is worth noticing, that only the $\Delta(1232)$ Dalitz decay was measured experimentally at the pole $(W \simeq 1.232 \mathrm{GeV})$. Our estimate of the $\Delta(1232)$ Dalitz decay width is consistent with the result of HADES [15]. All estimates for the $B^{\prime} \rightarrow e^{+} e^{-} B$ Dalitz decays at the physical decuplet baryon mass $\left(M_{B^{\prime}}\right)$ are presented in Table VI. Excluding the $\Delta(1232)$, the remaining estimates are predictions to be tested by future experiments.

The $\Sigma^{* 0} \rightarrow e^{+} e^{-} \Lambda$ decay width was also estimated within the chiral perturbation theory combined with dispersion relations [45], obtaining a slightly larger value

\footnotetext{
${ }^{3}$ This effect can be better understood assuming that the meson cloud contributions to the transition form factors can, near $q^{2}=0$, be simulated by a multipole function $1 /\left(1+Q^{2} / \Lambda^{2}\right)^{n}$, where $n>2$ is an integer (the exact value is not important for the discussion) and $\Lambda^{2}$ is a cutoff of $Q^{2}$. One conclude, then, that the cutoff associated with the kaon cloud is smaller than the one associated to the pion, since the kaon cloud effects are suppressed more strongly than the pion cloud effects. The consequence of this relation between cutoffs is that the magnitude of the derivative of the kaon cloud multipole $\left(\propto 1 / \Lambda^{2}\right)$ at $q^{2}=0$ is larger than the magnitude of the derivative of the pion cloud multipole.
}

(3.0-3.4 keV). The inclusion of the kaon cloud effects in our framework can also increase our estimate.

\section{OUTLOOK AND CONCLUSIONS}

The HADES facility provides a rare opportunity to study electromagnetic transitions between baryon states in the timelike region $\left(q^{2}>0\right)$. Those experiments complement the information obtained from electro-production of baryon resonances in the spacelike region $\left(q^{2} \leq 0\right)$. The recent and the upcoming results from HADES motivate the development of theoretical models for the $\gamma^{*} B \rightarrow B^{\prime}$ transition form factors in the timelike region, where $B$ and $B^{\prime}$ are generic baryons.

Of particular interest are the Dalitz decays of baryons $\left(B^{\prime} \rightarrow e^{+} e^{-} B\right)$, including hyperons. Measurements of the $\Delta(1232)$ Dalitz decays have been reported recently. The analysis of the $\Sigma^{0}(1385) \rightarrow e^{+} e^{-} \Lambda(1116)$ decay is expected in a near future. Due to the capability of HADES to produce hyperons, other decuplet baryon Dalitz decays are expected to be measured in the following years. The next natural candidate, based on the estimated magnitude, is the $\Sigma^{+}(1385) \rightarrow e^{+} e^{-} \Sigma^{+}(1193)$ decay.

To complement the experimental activity at HADES, we present here model estimates for the Dalitz decay rates and Dalitz decay widths for all decuplet baryons. Our calculations are based on the covariant spectator quark model for the octet baryon to decuplet baryon electromagnetic transitions, extended in the present work to the timelike region. The model was previously calibrated by lattice QCD data for the baryon octet and baryon decuplet, and takes into account the pion cloud dressing of the baryon cores. The model is successful in the description of the radiative decays: $\Delta(1232) \rightarrow \gamma N, \Sigma^{0}(1385) \rightarrow \gamma \Lambda(1116)$ and $\Sigma^{+}(1385) \rightarrow$ $\gamma \Sigma^{+}(1193)$. Under study is the extension of the present model with the inclusion of the kaon cloud contribution for finite $q^{2}$, which may approach the model estimates to the data.

We conclude that, in general, the valence quark effects give the dominant contribution to the transition form factors and to the Dalitz decay widths, but that pion cloud contribution provides significant corrections, which improve the description of the data. In most cases, the pion cloud effects contribute with about $30 \%-45 \%$ to the transition form factors near $q^{2}=0$. In some cases, those contributions are only about $20 \%\left(\Sigma^{+}(1385), \Sigma^{0}(1385)\right.$ and $\Xi^{0}(1530)$ decays $)$.

We conclude also, that different magnitudes are expected to the radiative and Dalitz decay widths according with valence quark content: large magnitudes for the $\Delta(1232)$, $\Sigma^{0}(1385) \rightarrow e^{+} e^{-} \Lambda(1116), \quad \Sigma^{+}(1385) \quad$ and $\quad \Xi^{0}(1530)$ decays; intermediate magnitudes for the $\Sigma^{0}(1385) \rightarrow$ $e^{+} e^{-} \Sigma^{0}(1193)$ decay; small magnitudes for the $\Sigma^{-}(1385)$ and $\Xi^{-}(1530)$ decays. We observed also that the $\Sigma^{+}(1385)$ and $\Xi^{0}(1530)$ decays, as well as the $\Sigma^{-}(1385)$ and $\Xi^{-}(1530)$ decays, have similar Dalitz decay rates. 
We also analyze the role of the $q^{2}$-dependence of the form factors. We conclude that, in general, the QED approach (constant form factor model) is not a good approximation, as already observed in the case of the $\Delta(1232)$ Dalitz decay. The impact of the $q^{2}$-dependence of the form factors is, however, less significant than in the case of the $\Delta(1232)$. The $\Sigma^{0}(1385) \rightarrow \gamma^{*} \Lambda(1116)$ transition form factors are enhanced in the timelike region due to the pion cloud effects. The $q^{2}$-dependence is also relevant for the $\Sigma^{-}(1385) \rightarrow e^{+} e^{-} \Sigma^{-}(1193)$ and $\Xi^{-}(1530) \rightarrow$ $e^{+} e^{-} \Xi^{-}(1318)$ decays.

The covariant spectator quark model proved also to be a useful framework to study Dalitz decays of nucleon excited states $\left(N^{*}\right)$, more specifically in the cases of the $\Delta(1232)$, $N(1520)$ and $N(1535)$ resonances [30-32]. Under study is the possibility of extending the formalism to other baryon systems, which may also be regarded as a combination of valence quark cores combined with meson cloud excitations of the baryon cores.

\section{ACKNOWLEDGMENTS}

G. R. thanks Piotr Salabura for helpful discussions and suggestions. G. R. was supported by the Fundação de Amparo à Pesquisa do Estado de São Paulo (FAPESP): Project No. 2017/02684-5, Grant No. 2017/17020-BCO-JP.

\section{APPENDIX A: QUARK FORM FACTORS}

Motivated by the VMD mechanism, we use the following parametrizations for the quark form factors $f_{i 0}$ and $f_{i \pm}$ $(i=1,2)$

$$
\begin{aligned}
& f_{1-}\left(q^{2}\right)=\lambda_{q}+\left(1-\lambda_{q}\right) \frac{m_{\rho}^{2}}{m_{\rho}^{2}-q^{2}}-c_{-} \frac{M_{h}^{2} q^{2}}{\left(M_{h}^{2}-q^{2}\right)^{2}} \\
& f_{1+}\left(q^{2}\right)=\lambda_{q}+\left(1-\lambda_{q}\right) \frac{m_{\omega}^{2}}{m_{\omega}^{2}-q^{2}}-c_{+} \frac{M_{h}^{2} q^{2}}{\left(M_{h}^{2}-q^{2}\right)^{2}} \\
& f_{10}\left(q^{2}\right)=\lambda_{q}+\left(1-\lambda_{q}\right) \frac{m_{\phi}^{2}}{m_{\phi}^{2}-q^{2}}-c_{0} \frac{M_{h}^{2} q^{2}}{\left(M_{h}^{2}-q^{2}\right)^{2}} \\
& f_{2-}\left(q^{2}\right)=\kappa_{-}\left\{d_{-} \frac{m_{\rho}^{2}}{m_{\rho}^{2}-q^{2}}+\left(1-d_{-}\right) \frac{M_{h}^{2}}{M_{h}^{2}-q^{2}}\right\} \\
& f_{2+}\left(q^{2}\right)=\kappa_{+}\left\{d_{+} \frac{m_{\omega}^{2}}{m_{\omega}^{2}-q^{2}}+\left(1-d_{+}\right) \frac{M_{h}^{2}}{M_{h}^{2}-q^{2}}\right\} \\
& f_{20}\left(q^{2}\right)=\kappa_{0}\left\{d_{0} \frac{m_{\phi}^{2}}{m_{\phi}^{2}-q^{2}}+\left(1-d_{0}\right) \frac{M_{h}^{2}}{M_{h}^{2}-q^{2}}\right\}
\end{aligned}
$$

where $m_{\rho}, m_{\omega}$ and $m_{\phi}$ represent the masses of the mesons $\rho, \omega$ and $\phi$, respectively. The terms with $M_{h}$ correspond to an effective heavy vector meson which parametrize the short range effects. The value of $M_{h}$ is fixed as $M_{h}=2 M_{N}$ $[76,86]$. In numerical calculations, we use the approximation $m_{\omega}=m_{\rho}$ for simplicity.

In Eqs. (A4)-(A6), $\kappa_{q}$ represent quark anomalous magnetic moments. We use the parametrization derived from the study of the octet and decuplet baryons $[10,77]$. We take in particular $\kappa_{-}=1.435, \kappa_{+}=1.803$ and $\kappa_{0}=1.462$. To convert to the flavors $q=u, d, s$, one uses $\kappa_{u}=\frac{1}{4}\left(\kappa_{+}+3 \kappa_{-}\right), \kappa_{d}=\frac{1}{2}\left(2 \kappa_{-}-\kappa_{+}\right)$and $\kappa_{s}=\kappa_{0}$ [76,77].

In the equations $\lambda_{q}$ is a parameter related with the quark density number in deep inelastic scattering [76]. The numerical value is $\lambda_{q}=1.21$. The remaining parameters are $c_{+}=4.160, \quad c_{-}=1.160, \quad c_{0}=4.427, \quad d_{+}=d_{-}=$ -0.686 and $d_{0}=-1.860[10,77]$.

The expressions (A1)-(A6) are valid in the region $q^{2}<0$, when the vector meson decay widths vanish, $\Gamma_{v} \equiv$ $0(v=\rho, \omega, \phi)$. For the extension of the quark form factors to the timelike region $\left(q^{2}>0\right)$, we consider the replacement $(v=\rho, \omega, \phi)$

$$
\frac{m_{v}^{2}}{m_{v}^{2}-q^{2}} \rightarrow \frac{m_{v}^{2}}{m_{v}^{2}-q^{2}-i m_{v} \Gamma_{v}\left(q^{2}\right)} .
$$

The decay width functions $\Gamma_{v}\left(q^{2}\right)$, which describe the dressing of the vector mesons in terms of the possible meson decay channels, are discussed next.

Following our previous works based on the $\Delta(1232)$ Dalitz decay, we consider for the isovector components ( $\rho$-pole) the function $[30,75,99,107]$

$$
\Gamma_{\rho}\left(q^{2}\right)=\Gamma_{\rho}^{0} \frac{m_{\rho}^{2}}{q^{2}}\left(\frac{q^{2}-4 m_{\pi}^{2}}{m_{\rho}^{2}-4 m_{\pi}^{2}}\right)^{3 / 2} \theta\left(q^{2}-4 m_{\pi}^{2}\right),
$$

where $\Gamma_{\rho}^{0}=0.149 \mathrm{GeV}$. The previous equation parametrize the width associated to the decay $\rho \rightarrow 2 \pi$ for a virtual $\rho$ with square four-momentum $q^{2}[107,108]$. Alternative parametrizations for $\Gamma_{\rho}\left(q^{2}\right)$ are presented in Refs. [19,109-111].

For the isoscalar channel, associated with the $\omega$-meson, one needs to consider the combination of the decays $\omega \rightarrow 2 \pi$ and $\omega \rightarrow 3 \pi$. Following our work on the $N(1520)$ Dalitz decay [31], we decompose

$$
\Gamma_{\omega}\left(q^{2}\right)=\Gamma_{2 \pi}\left(q^{2}\right)+\Gamma_{3 \pi}\left(q^{2}\right),
$$

where the first term parametrize the decay $\omega \rightarrow 2 \pi$ a and the second term parametrize the decay $\omega \rightarrow 3 \pi$. The expression for $\Gamma_{2 \pi}\left(q^{2}\right)$ is similar to $\Gamma_{\rho}\left(q^{2}\right)$ except for the strength [31,107]. As for the decay $\omega \rightarrow 3 \pi$, we consider a model based on the process $\omega \rightarrow \rho \pi \rightarrow 3 \pi$, where the intermediate $\rho$ decays into 2 pions [107]. We do not reproduce here the expressions for $\Gamma_{2 \pi}$ and $\Gamma_{3 \pi}$, since they can be found in Ref. [31]. We just point out that the $3 \pi$ 
channel dominates for $q^{2}>0.55 \mathrm{GeV}^{2}$. A more detailed discussion of $\Gamma_{\omega}\left(q^{2}\right)$ is presented in Ref. [31].

Finally, for the $\phi$ decay width, we consider the simplified parametrization

$\Gamma_{\phi}\left(q^{2}\right)=\Gamma_{\phi}^{0} \frac{m_{\phi}^{2}}{q^{2}}\left(\frac{q^{2}-4 m_{K}^{2}}{m_{\phi}^{2}-4 m_{K}^{2}}\right)^{3 / 2} \theta\left(q^{2}-4 m_{K}^{2}\right)$,

where $\Gamma_{\phi}^{0}=4.23 \times 10^{-3} \mathrm{GeV}$, and $m_{K}$ is the kaon mass $\left(m_{K} \simeq 0.5 \mathrm{GeV}\right)$. Equation (A10) describes the $\phi \rightarrow 2 K(K$ is the kaon) under the assumption that it is the dominate decay of the $\phi$. According with PDG the $2 K$ decays correspond to about $85 \%$ of the $\phi$ decays [6].

For the range of the calculation of the present work $(W<2 \mathrm{GeV})$ the regularization of the $\phi$ pole is not very relevant, since $m_{\phi}^{2} \simeq 1 \mathrm{GeV}^{2} \gg q^{2}$. The singularities associated to the $\phi$-meson appear, then only for $W \geq M_{B}+m_{\phi}>2.1 \mathrm{GeV}$. Nevertheless, we regularize the $\phi$-propagator for consistence. We note also that even the calculations more dependent on the $\phi$-pole, in particular the Dalitz decay widths $\Gamma_{e^{+} e^{-} B}(W)$, are weakly dependent on the shape of $\Gamma_{\phi}\left(q^{2}\right)$.

We also concluded that the Dalitz decay widths $\Gamma_{e^{+} e^{-B}}(W)$ depend weakly of the explicit form used for $\Gamma_{\rho}\left(q^{2}\right)$ in Eq. (A8). Equivalent results can be obtained when we replace $\frac{m_{\rho}^{2}}{q^{2}}$ by $\frac{m_{\rho}}{q}$, following Refs. [109]. The main differences appear only near $q^{2}=m_{\rho}^{2}$, and their effects are diluted in the integration in $q$.

\section{APPENDIX B: REGULARIZATION OF HIGH MASS POLES}

For a given $W$ the square momentum $q^{2}$ is limited by the kinematic condition $q^{2} \leq\left(W-M_{B}\right)^{2}$. If there is a singularity at $q^{2}=\Lambda^{2}$ the singularity will appear for values of $W$ such that $\left(W-M_{B}\right)^{2} \geq q^{2}$, or $W \geq M_{B}+\Lambda$.

To avoid those singularities, for single poles with a generic momentum scale $\Lambda$, we use the following procedure

$$
\frac{\Lambda^{2}}{\Lambda^{2}-q^{2}} \rightarrow \frac{\Lambda^{2}}{\Lambda^{2}-q^{2}-i \Lambda \Gamma_{X}\left(q^{2}\right)},
$$

where

$$
\Gamma_{X}\left(q^{2}\right)=4 \Gamma_{X}^{0}\left(\frac{q^{2}}{q^{2}+\Lambda^{2}}\right)^{2} \theta\left(q^{2}\right) .
$$

In the last equation $\Gamma_{X}^{0}$ is a constant given by $\Gamma_{X}^{0}=4 \Gamma_{\rho}^{0} \simeq 0.6 \mathrm{GeV}$.

This procedure is used on the pole $q^{2}=M_{h}^{2}$ of the quarks form factors, for the single pole (Pauli form factors) and double pole (Dirac form factors).
For powers of monopole factors used in the pion cloud contribution (3.21), we approximate the result by the magnitude of the expression:

$$
\left(\frac{\Lambda^{2}}{\Lambda^{2}-q^{2}}\right)^{n} \rightarrow\left(\frac{\Lambda^{4}}{\left(\Lambda^{2}-q^{2}\right)^{2}+\Lambda^{2}\left[\Gamma_{X}\left(q^{2}\right)\right]^{2}}\right)^{\frac{n}{2}},
$$

where $\Gamma_{X}\left(q^{2}\right)$ is determined by Eq. (B2).

In the generalization of the dipole function $\tilde{G}_{D}$ defined by Eq. (3.24), we use Eq. (B2), with $\Lambda=\Lambda_{D}$.

\section{APPENDIX C: CALCULATION OF THE MESON CLOUD CONTRIBUTIONS}

We present here a brief revision of the calculation of the contributions of the diagrams (a) and (b) from Fig. 1, following Ref. [13].

The calculations of the meson cloud contributions are based on the cloudy bag model (CBM). Since those contributions depend on the photon couplings with the bare baryons, it is necessary to make the connection between the Dirac and Pauli couplings between CBM and the covariant spectator quark model. This connection was performed in Ref. [13] with the comparison of the results from both frameworks for the octet baryon to decuplet baryon transitions. One obtains the same result for the magnetic transition form factor at low $Q^{2}$ in both frameworks, when we define the quark $(q=u, d, s)$ effective magnetic moments as

$$
\mu_{q}=\sqrt{\frac{2}{3}}\left[\frac{2 M_{B}}{M_{B^{\prime}}+M_{B}}+\frac{M_{B}}{M_{N}} \kappa_{q}\right] \mathcal{I}\left(0, M_{B^{\prime}}\right),
$$

where $\mathcal{I}\left(0, M_{B^{\prime}}\right)$ is defined by Eq. (3.10). Notice that the value of $\mu_{q}$ depends on the explicit transition. In the static limit, where all baryons are very heavy and the mass differences can be neglected, one obtains $\mu_{q} \propto\left(1+\kappa_{q}\right)$.

In Eq. (C1), the presence of the overlap integral is important because it tend to reduce the contribution of the bare core when we use different radial wave functions for the octet baryon and decuplet baryon. In an exact $S U(3)$ model where octet and decuplet baryons are described by the same radial wave functions (also no mass difference), we obtain $\mathcal{I}\left(0, M_{B^{\prime}}\right)=1$ [13].

We can now describe the calculations of the meson cloud contributions from the diagrams Fig. 1(a) and (b) to the magnetic form factors.

\section{Diagram (a)}

The calculation of the contributions for the diagram 1(a) are performed based on

$$
G_{M}^{\mathrm{MC} a}=\sum_{M, B_{1}} C_{B B^{\prime} ; B_{1}}^{M} H_{B B^{\prime}}^{M}\left(B_{1}\right),
$$


TABLE VII. Diagram a: $B_{1}$ contributions for the $B^{\prime} \rightarrow \gamma^{*} B$ decay. There are contributions for $M=\pi$ and $M=K$.

\begin{tabular}{lcc}
\hline \hline & $\pi$ & $K$ \\
\hline$\Delta \rightarrow \gamma^{*} N$ & $N, \Delta$ & $\Sigma, \Sigma^{*}$ \\
$\Sigma^{* 0} \rightarrow \gamma^{*} \Lambda$ & $\Sigma, \Sigma^{*}$ & $N, \Xi, \Xi^{*}$ \\
$\Sigma^{*} \rightarrow \gamma^{*} \Sigma$ & $\Lambda, \Sigma, \Sigma^{*}$ & $N, \Delta, \Xi, \Xi^{*}$ \\
$\Xi^{*} \rightarrow \gamma^{*} \Xi$ & $\Xi, \Xi^{*}$ & $\Lambda, \Sigma, \Sigma^{*}, \Omega$ \\
\hline \hline
\end{tabular}

where $M$ labels the intermediate meson states $(M=\pi, K)$, $C_{B B^{\prime} ; B_{1}}^{M}$ are coefficients in the CBM framework, and $H_{B B^{\prime}}^{M}\left(B_{1}\right)$ is the CBM loop integral associated to a diagram with an intermediate baryon $B_{1}$ and the meson $M$. The function $H_{B B^{\prime}}^{M}\left(B_{1}\right)$ is defined by Eq. (4.2) from Ref. [13].

The labels of the state $B_{1}$ used in the calculations are displayed in Table VII. The couplings associated to the states are presented in Table IV from Ref. [13]. For completeness, we present also the intermediate states associated to the kaon.

\section{Diagram (b)}

The calculations of the contributions for the diagram 1(b) are performed based on

$$
G_{M}^{\mathrm{MC} b}=\sum_{M, B_{1}, B_{2}} D_{B B^{\prime} ; B_{1}, B_{2}}^{M} H_{B B^{\prime}}^{2 M}\left(B_{1}, B_{2}\right),
$$

where $M$ labels the intermediate meson states $(M=\pi, K$, $\eta), D_{B B^{\prime} ; B_{1}, B_{2}}^{M}$ are coefficients in the CBM framework, and $H_{B B^{\prime}}^{2 M}\left(B_{1}, B_{2}\right)$ is the CBM loop integral associated to a diagram with the intermediate baryons $B_{1}, B_{2}$ and the
TABLE VIII. Diagram (b): contributions $B^{\prime} B_{1} B_{2} B$ for the $B^{\prime} \rightarrow$ $\gamma^{*} B$ decay. There are contributions for $M=\pi, M=K$ and $M=\eta$.

\begin{tabular}{cccc}
\hline \hline & $\pi$ & $K$ & $\eta$ \\
\hline$\Delta \rightarrow \gamma^{*} N$ & $N N, N \Delta$ & $\Lambda \Sigma, \Lambda \Sigma^{*}, \Sigma \Sigma$ & $N \Delta$ \\
& $\Delta N, \Delta \Delta$ & $\Sigma \Sigma^{*}, \Sigma^{*} \Sigma, \Sigma^{*} \Sigma^{*}$ & \\
$\Sigma^{* 0} \rightarrow \gamma^{*} \Lambda$ & $\Sigma \Lambda, \Sigma \Sigma, \Sigma \Sigma^{*}$ & $N N, N \Delta$ & $\Lambda \Sigma$ \\
& $\Sigma^{*} \Lambda, \Sigma^{*} \Sigma, \Sigma^{*} \Sigma^{*}$ & $\Xi \Xi, \Xi \Xi^{*}, \Xi^{*} \Xi, \Xi^{*} \Xi^{*}$ & \\
$\Sigma^{*} \rightarrow \gamma^{*} \Sigma$ & $\Sigma \Lambda, \Sigma \Sigma, \Sigma \Sigma^{*}$ & $N N, N \Delta, \Delta N, \Delta \Delta$ & $\Sigma \Sigma, \Sigma^{*} \Sigma$ \\
& $\Lambda \Lambda, \Lambda \Sigma, \Lambda \Sigma^{*}$ & $\Xi \Xi, \Xi \Xi^{*}, \Xi^{*} \Xi, \Xi^{*} \Xi^{*}$ \\
& $\Sigma^{*} \Lambda, \Sigma^{*} \Sigma, \Sigma^{*} \Sigma^{*}$ & & \\
$\Xi^{*} \rightarrow \gamma^{*} \Xi$ & $\Xi \Xi, \Xi \Xi^{*}$ & $\Sigma \Sigma, \Sigma \Sigma^{*}, \Sigma^{*} \Sigma, \Sigma^{*} \Sigma^{*}$ & $\Xi \Xi, \Xi \Xi^{*}$ \\
& $\Xi^{*} \Xi, \Xi^{*} \Xi^{*}$ & $\Lambda \Lambda, \Lambda \Sigma, \Lambda \Sigma^{*}$ & $\Xi^{*} \Xi, \Xi^{*} \Xi^{*}$ \\
& \multicolumn{3}{c}{$\Sigma \Lambda, \Sigma^{*} \Lambda, \Omega \Omega$} \\
\hline \hline
\end{tabular}

meson $M$. The integral $H_{B B^{\prime}}^{2 M}\left(B_{1}, B_{2}\right)$ is defined by Eq. (4.4) in Ref. [13].

The function $G_{M}^{\mathrm{MC} b}$ include the contributions of the baryons $B_{1}, B_{2}$ displayed in Table VIII. The explicit expressions for $D_{B B^{\prime} ; B_{1}, B_{2}}^{M}$ are linear combinations of the effective quark form factors $\mu_{q}$ and are presented in the Appendix A of Ref. [13].

The dependence of the diagram 1(b) contributions on the intermediate bare states are then expressed by the dependence on the effective quark form factors.

Note that in intermediate state, one has all kinds of baryon transitions: octet to octet, octet to decuplet, decuplet to octet and decuplet to decuplet.
[1] I. G. Aznauryan et al., Int. J. Mod. Phys. E 22, 1330015 (2013).

[2] I. G. Aznauryan and V. D. Burkert, Prog. Part. Nucl. Phys. 67, 1 (2012).

[3] G. Ramalho, Few-Body Syst. 59, 92 (2018).

[4] V. D. Burkert and T. S. H. Lee, Int. J. Mod. Phys. E 13, 1035 (2004).

[5] D. Drechsel, S. S. Kamalov, and L. Tiator, Eur. Phys. J. A 34, 69 (2007).

[6] M. Tanabashi et al. (Particle Data Group), Phys. Rev. D 98, 030001 (2018).

[7] G. Ramalho, M. T. Peña, and K. Tsushima, Phys. Rev. D 101, 014014 (2020).

[8] F. Gross, G. Ramalho, and K. Tsushima, Phys. Lett. B 690, 183 (2010).

[9] G. Ramalho and K. Tsushima, Phys. Rev. D 84, 054014 (2011).
[10] G. Ramalho, K. Tsushima, and A. W. Thomas, J. Phys. G 40, 015102 (2013); G. Ramalho, J. P. B. C. de Melo, and K. Tsushima, Phys. Rev. D 100, 014030 (2019).

[11] G. Ramalho and K. Tsushima, Phys. Rev. D 86, 114030 (2012).

[12] G. Ramalho and K. Tsushima, Phys. Rev. D 87, 093011 (2013).

[13] G. Ramalho and K. Tsushima, Phys. Rev. D 88, 053002 (2013).

[14] G. Agakishiev et al., Eur. Phys. J. A 50, 82 (2014).

[15] J. Adamczewski-Musch et al. (HADES Collaboration), Phys. Rev. C 95, 065205 (2017).

[16] J. Adamczewski-Musch et al. (HADES Collaboration), Eur. Phys. J. A 53, 149 (2017).

[17] P. Salabura et al. (HADES Collaboration), J. Phys. Conf. Ser. 420, 012013 (2013). 
[18] P. Cole, B. Ramstein, and A. Sarantsev, Few-Body Syst. 59, 144 (2018).

[19] J. Weil, H. van Hees, and U. Mosel, Eur. Phys. J. A 48, 111 (2012); 48, 150(E) (2012).

[20] B. Ramstein (HADES Collaboration), Few-Body Syst. 59, 141 (2018).

[21] J. Adamczewski-Musch et al. (HADES Collaboration), Phys. Rev. C 102, 024001 (2020).

[22] P. Salabura and J. Stroth, arXiv:2005.14589.

[23] B. Ramstein, Few-Body Syst. 59, 143 (2018).

[24] J. Adamczewski-Musch et al. (HADES Collaboration), Eur. Phys. J. A 53, 188 (2017).

[25] B. Ramstein et al. (HADES Collaboration), EPJ Web Conf. 199, 01008 (2019).

[26] M. F. M. Lutz, B. Friman, and M. Soyeur, Nucl. Phys. A713, 97 (2003).

[27] R. Shyam and U. Mosel, Phys. Rev. C 82, 062201(R) (2010).

[28] M. Zetenyi and G. Wolf, Phys. Rev. C 86, 065209 (2012).

[29] F. Dohrmann et al., Eur. Phys. J. A 45, 401 (2010).

[30] G. Ramalho, M. T. Peña, J. Weil, H. van Hees, and U. Mosel, Phys. Rev. D 93, 033004 (2016).

[31] G. Ramalho and M. T. Peña, Phys. Rev. D 95, 014003 (2017).

[32] G. Ramalho and M. T. Peña, Phys. Rev. D 101, 114008 (2020).

[33] W. Przygoda (HADES Collaboration), J. Phys. Soc. Jpn. Conf. Proc. 10, 010013 (2016).

[34] F. Scozzi (HADES Collaboration), EPJ Web Conf. 137, 05023 (2017).

[35] W. J. Briscoe, M. Doring, H. Haberzettl, D. M. Manley, M. Naruki, I. I. Strakovsky, and E. S. Swanson, Eur. Phys. J. A 51, 129 (2015).

[36] S. Pacetti, R. Baldini Ferroli, and E. Tomasi-Gustafsson, Phys. Rep. 550-551, 1 (2015).

[37] B. Aubert et al. (BABAR Collaboration), Phys. Rev. D 76, 092006 (2007).

[38] S. Dobbs, K. K. Seth, A. Tomaradze, T. Xiao, and G. Bonvicini, Phys. Rev. D 96, 092004 (2017).

[39] M. Ablikim et al. (BESIII Collaboration), Phys. Rev. D 97, 032013 (2018).

[40] B. Singh et al. (PANDA Collaboration), Phys. Rev. D 95, 032003 (2017).

[41] G. Ramalho (to be published).

[42] R. Lalik (HADES Collaboration), J. Phys. Conf. Ser. 1137, 012057 (2019).

[43] N. Rathod, R. Lalik, A. Malige, P. Salabura, and J. Smyrski, Acta Phys. Pol. B 51, 239 (2020).

[44] HADES Collaboration (to be published).

[45] O. Junker, S. Leupold, E. Perotti, and T. Vitos, Phys. Rev. C 101, 015206 (2020).

[46] M. Holmberg and S. Leupold, Eur. Phys. J. A 54, 103 (2018).

[47] E. Kaxiras, E. J. Moniz, and M. Soyeur, Phys. Rev. D 32, 695 (1985).

[48] R. A. Williams, C. R. Ji, and S. R. Cotanch, Phys. Rev. C 48, 1318 (1993).

[49] J. J. Sakurai, Ann. Phys. (N.Y.) 11, 1 (1960).

[50] M. I. Krivoruchenko, B. V. Martemyanov, A. Faessler, and C. Fuchs, Ann. Phys. (Amsterdam) 296, 299 (2002).

[51] F. Iachello and Q. Wan, Phys. Rev. C 69, 055204 (2004).
[52] G. Ramalho, Phys. Rev. D 100, 114014 (2019).

[53] G. Ramalho, Eur. Phys. J. A 54, 75 (2018); Phys. Rev. D 94, 114001 (2016).

[54] G. Ramalho, Phys. Rev. D 93, 113012 (2016).

[55] G. Ramalho, Eur. Phys. J. A 55, 32 (2019).

[56] R. K. Sahoo, A. R. Panda, and A. Nath, Phys. Rev. D 52, 4099 (1995).

[57] G. Wagner, A. J. Buchmann, and A. Faessler, Phys. Rev. C 58, 1745 (1998).

[58] R. Bijker, F. Iachello, and A. Leviatan, Ann. Phys. (Berlin) 284, 89 (2000).

[59] D. Keller et al. (CLAS Collaboration), Phys. Rev. D 85, 059903 (2012).

[60] J. W. Darewych, M. Horbatsch, and R. Koniuk, Phys. Rev. D 28, 1125 (1983).

[61] H. Sanchis-Alepuz and C. S. Fischer, Eur. Phys. J. A 52, 34 (2016).

[62] H. Sanchis-Alepuz, R. Alkofer, and C. S. Fischer, Eur. Phys. J. A 54, 41 (2018).

[63] D. B. Leinweber, T. Draper, and R. M. Woloshyn, Phys. Rev. D 48, 2230 (1993).

[64] T. M. Aliev and A. Ozpineci, Nucl. Phys. B732, 291 (2006).

[65] L. Wang and F.X. Lee, Phys. Rev. D 80, 034003 (2009).

[66] C. L. Schat, C. Gobbi, and N. N. Scoccola, Phys. Lett. B 356, 1 (1995).

[67] T. Haberichter, H. Reinhardt, N. N. Scoccola, and H. Weigel, Nucl. Phys. A615, 291 (1997).

[68] J. Y. Kim and H. C. Kim, arXiv:2002.05980.

[69] M. N. Butler, M. J. Savage, and R. P. Springer, Nucl. Phys. B399, 69 (1993).

[70] D. Arndt and B. C. Tiburzi, Phys. Rev. D 69, 014501 (2004).

[71] R. F. Lebed and R. H. TerBeek, Phys. Rev. D 83, 016009 (2011).

[72] G. Ramalho, M. T. Peña, and F. Gross, Eur. Phys. J. A 36, 329 (2008).

[73] G. Ramalho, M. T. Peña, and F. Gross, Phys. Rev. D 78, 114017 (2008).

[74] G. Ramalho and M. T. Peña, Phys. Rev. D 80, 013008 (2009).

[75] G. Ramalho and M. T. Peña, Phys. Rev. D 85, 113014 (2012).

[76] F. Gross, G. Ramalho, and M. T. Peña, Phys. Rev. C 77, 015202 (2008).

[77] G. Ramalho, K. Tsushima, and F. Gross, Phys. Rev. D 80, 033004 (2009).

[78] F. Gross, G. Ramalho, and M. T. Peña, Phys. Rev. D 85, 093005 (2012).

[79] G. Ramalho and K. Tsushima, Phys. Rev. D 94, 014001 (2016).

[80] G. Ramalho and K. Tsushima, Phys. Rev. D 82, 073007 (2010).

[81] G. Ramalho, D. Jido, and K. Tsushima, Phys. Rev. D 85, 093014 (2012).

[82] G. Ramalho and K. Tsushima, Phys. Rev. D 81, 074020 (2010); 89, 073010 (2014).

[83] G. Ramalho and M. T. Peña, Phys. Rev. D 95, 014003 (2017); 84, 033007 (2011). 
[84] G. Ramalho and M. T. Peña, J. Phys. G 36, 085004 (2009); G. Ramalho, M. T. Peña, and F. Gross, Phys. Rev. D 81, 113011 (2010); G. Ramalho and M. T. Peña, Phys. Rev. D 83, 054011 (2011).

[85] G. Ramalho, Phys. Rev. D 95, 054008 (2017); 90, 033010 (2014).

[86] G. Ramalho and M. T. Peña, J. Phys. G 36, 115011 (2009).

[87] M. I. Krivoruchenko and A. Faessler, Phys. Rev. D 65, 017502 (2001).

[88] G. Wolf, G. Batko, W. Cassing, U. Mosel, K. Niita, and M. Schaefer, Nucl. Phys. A517, 615 (1990).

[89] H. F. Jones and M. D. Scadron, Ann. Phys. (N.Y.) 81, 1 (1973).

[90] R. C. E. Devenish, T. S. Eisenschitz, and J. G. Korner, Phys. Rev. D 14, 3063 (1976).

[91] F. Gross, Phys. Rev. 186, 1448 (1969); A. Stadler, F. Gross, and M. Frank, Phys. Rev. C 56, 2396 (1997).

[92] C. E. Carlson and N. C. Mukhopadhyay, Phys. Rev. Lett. 81, 2646 (1998); C. E. Carlson, Phys. Rev. D 34, 2704 (1986); Few-Body Syst. Suppl. 11, 10 (1999).

[93] H. W. Lin and K. Orginos, Phys. Rev. D 79, 074507 (2009).

[94] S. Boinepalli, D. B. Leinweber, P. J. Moran, A. G. Williams, J. M. Zanotti, and J. B. Zhang, Phys. Rev. D 80, 054505 (2009).

[95] B. Julia-Diaz, T.-S. H. Lee, T. Sato, and L. C. Smith, Phys. Rev. C 75, 015205 (2007).

[96] A. W. Thomas, Adv. Nucl. Phys. 13, 1 (1984).

[97] S. Theberge and A. W. Thomas, Nucl. Phys. A393, 252 (1983).
[98] T. Yamaguchi, K. Tsushima, Y. Kohyama, and K. Kubodera, Nucl. Phys. A500, 429 (1989).

[99] H. C. Donges, M. Schafer, and U. Mosel, Phys. Rev. C 51, 950 (1995).

[100] C. Hanhart, Phys. Lett. B 715, 170 (2012).

[101] M. Herrmann, B. L. Friman, and W. Norenberg, Nucl. Phys. A560, 411 (1993).

[102] K. Nakamura et al. (Particle Data Group), J. Phys. G 37, 075021 (2010).

[103] D. Keller et al. (CLAS Collaboration), Phys. Rev. D 83, 072004 (2011).

[104] J. Colas, C. Farwell, A. Ferrer, and J. Six, Nucl. Phys. B91, 253 (1975).

[105] V. V. Molchanov et al. (SELEX Collaboration), Phys. Lett. B 590, 161 (2004).

[106] M. Ablikim et al., Phys. Rev. D 101, 012004 (2020).

[107] P. Mühlich and U. Mosel, Nucl. Phys. A773, 156 (2006); P. Mühlich, Ph. D. thesis, University of Giessen, 2007, https://inspirehep.net/literature/1375870.

[108] H. B. O'Connell, B. C. Pearce, A. W. Thomas, and A. G. Williams, Prog. Part. Nucl. Phys. 39, 201 (1997).

[109] G. J. Gounaris and J. J. Sakurai, Phys. Rev. Lett. 21, 244 (1968); H. B. O'Connell, B. C. Pearce, A. W. Thomas, and A. G. Williams, Phys. Lett. B 354, 14 (1995).

[110] M. Benayoun, M. Feindt, M. Girone, A. Kirk, P. Leruste, J. L. Narjoux, and K. Safarik, Z. Phys. C 58, 31 (1993).

[111] D. M. Manley and E. M. Saleski, Phys. Rev. D 45, 4002 (1992). 